\title{
INJECTION AND PROPAGATION OF A NONRELATIVISTIC \\ ELECTRON BEAM AND SPACECRAFT CHARGING
}

PPPL -2438

H. Okuda and J. Berchem

DE87 013206

\author{
Plasma Physics Laboratory, Princeton University \\ Princeton, New Jersey OB544, U.S.A.
}

\begin{abstract}
Two-dimensional numerical simulations have been carried out in order to study the injection and propagation of a nonrelativistic electron beam from a spacecraft into a fully ionized plasma in a magnetic field. Contrary to the earlier results in one-dimension, a high density electron beam whose density is comparable to the ambient density can propagate into a plasma. A strong radial electric field resulting from the net charges in the beam causes the beam electrons to spread radially reducing the beam density. When the injection current exceeds the return current, significant changing of the spacecraft is observed along with the reflection of the injected electrons back to the spacecraft. Recent data on the electron beam injection from the Spacelab 1 (SEPAC) are discussed.
\end{abstract}

\section{DISCLAIMER}

This report was prepared as an account of work sponsored by an agency of the United States

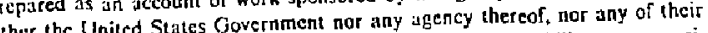
Government. Neither the United stess or implicd, or assumes any legal liability or responsiemployecs, makes any biastant, express or implied, of ass informaticn, apparatus, product. or bility for the accuracy, compleleness, or usefulness of any information, laphed rights. Referpresess disclosed, or represents that its use would not infringe prive by trade name, tradematk,

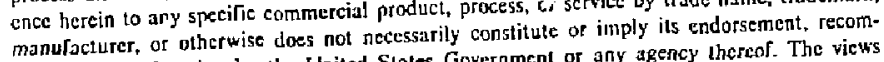
merdation, or lavoring by the United States Government or any agency theren. The the and opinions of authors expressed hercin do not

United States Government or any agency thereof. 


\section{Introduction}

A number of artificial particle beam experiments have been carried out in the last decade using sounding rockets and artificial satellites (Grandal, 1982). Most of such active experiments are carried out in order to study the properties of the ionospheric and magnetospheric plasmas and magnetic field. In more recent years, as the power of the electron beam becomes large, such artificial beams can be used to actively modify the ambient space plasmas. One such example is the SEPAC experiment carried out on board the Spacelab 1 in which an intense electron beam was injected into the ionosphere in an attempt to produce artificial auroral discharges. (Kawashima, 1982; Obayashi et al. , 1984).

As the beam electrons become more energetic, it is very important to understand the interaction between beam paricicles and the ambient plasma, including spacecraft charging, beam-plasma instability, enhanced ionization of the surrounding gas, and radiation generation. These processes collectively determine the propagation of the injected beam into the ambient space plasma.

There are a number of one-dimensional studies on the injection and propagation of a nonrelativistic electron beam into a plasma (Okuda et al., 1987; Okuda and Kan, 1987). It was found that the electron beam can propagate into an ambient plasma freely so long as the beam density is much less than the ambient density. In particular, the injection current can exceed the return current to the spacecraft carried by the thermal electrons in an ambient plasma. This is because the electric field amising from the beam injection accelerates the ambient electrons toward the spacecraft cokuda et al., 1987). A strong beam-plasma instability is found to accelerate the beam and the ambient electrons beyond the initial beam speed thereby forming a high energy tail. A presence of such a high energy electron population can lead to 
the generation of an anomalous conductivity where the classical mean-free path after the beam-plasma instability may be longer than the mean-free path of the original beam electrons (Okuda et al., 1985).

When the beam density becomes comparable to the ambient density, beam propagation is prohibited due to the reflection of the injected beam electrons, causing a charging of a spacecraft.

These results found in one-dimensional simulation remain valid only in the presence of a strong axial magnetic field preventing the beam electrons from spreading radially. In two and three dimensions where the radial spread of an electron is allowed, the one-dimensional results at high beam densities may have to be modified, especially when the ambient magnetic field is not strong enough. Even in a completely neutralized beam, it is pointed out that the beam electrons suffer from radial diffusion much larger than the diffusion of the ambient electrons (Okuda, 1987). This is due to the resonance of the beam electrons "with the waves generated as a result of beam-plasma instability. The electrons experience a D.C. electric field, $w-k_{\Downarrow} v_{0} \approx 0$, resulting in a large radial excursion given by $\mathrm{cE} \times \mathrm{B} / \mathrm{B}^{2} \mathrm{drift}$, and the diffusion coefficient may be estimated by $\left(c^{2} E_{\theta}^{2} / B^{2}\right)_{Y}^{-1}$, where $E_{\theta}$ is the amplitude of the azimuthal electric field and $\gamma$ is the growth rate of the beam-plasma instability.

When the beam is not neutralized, such as in the case of beam infection from a spacecraft into the ionosphere, effects of non-neutrality and spacecraft charging must be included to determine the beam propagation and beam diffusion.

In this paper, we present results obtained from two-dimensional numerical plasma simulations in a uniform ambient magnetic field. Following the introduction, the simulation model is presented in sec. II. The results of 
simulations on the propagation of the beam electrons and spacecraft charging are given in Sec. III. In Sec. IV, data from the SEPAC experiments are compared with the results of the numerical simulations.

\section{Simulation Model}

Consider an electron beam injected into space from a spacecraft. The beam electrons are injected along a magnetic field at a constant rate in time at a given energy and density into a fully ionized ambient plasma. A spacecraft is assumed to be a perfect conductor located at $y \leq 0$ as shown in Fig. 1. The ambient plasma is initially uniform and has Maxwellian velocity distributions for both ions and electrons with $T_{e}=T_{i}$. We shall assume that the spacecraft is much larger than the dimension of an electron beam so that the spacecraft extends from $x=0$ to $x=L_{x}$. An electron beam with the width $L_{b}$ is infected from a localized area of a spacecraft into the ambient plasma which is charge neutral before injection.

The injection of an electron beam and the flux of the ambient electrons and ions into the spacecraf't determine the charging. As in the onedimensional simulation models, we shall assume the ambient electrons and lons impinging upon the spacecraft are lost from the simulations via recombination. The same is true for the beam electrons which come back to the spacecraft after reflection. Ambient particles are reflected at $y=L_{y}$ so as to maintain the initial uniform plasma density (Okuda et al., 1987). Periodic boundary conditions are employed at $x=0$ and $x=L_{x}$ boundaries. On the surface of the spacecraft, the electrostatic potential $\phi(x, y=0)=$ constant holds since the spacecraft is assumed a perfect conductor. At $y=L_{y}$, we shall assume $\phi\left(x, y=L_{y}\right)=0$ since the location is sufficiently away from the beam and the spacecraft for most of the time. This is an approximate boundary 
condition which is valid only for $L_{y}=x$; however, the choice of the other boundary conditions at $y=L_{y}$ such as $a p\left(x, y^{\prime}=L_{y}\right) / a x=0$ which assume that the electric field lines are parallel to $y$ at $y=L_{y}$ did not significantly change the results of the simulations. In the simulations, the potential is solved in the following manner: since $\phi(x, y)$ is assumed periodic in $x$, $o(x$, $y)=I_{k_{x}} s_{k_{x}}(y) \exp \left(i k_{x}\right)$. For the $k_{x}=0$ mode, we shall apply the same boundary condition as in our one-dimensional model (Okuda et al., 1987) so that $\phi_{k_{y}}\left(y=L_{y}\right)=\partial \phi_{k_{x}}\left(y=L_{y}\right) / a y=0$. For two-dimensional $k_{x}=0$ modes, $\Phi_{k_{x}}(y=0)=\rho_{k_{x}}\left(y=L_{y}\right)=0$ are used. In this way the spacecraft potential $\phi_{s}=$ $\phi(x, y=0)$ can be determined uniquely.

\section{Results of Simulations}

We shall first consider a case where the beam density $n_{b}$ is equal to the average ambient density $n_{0}$ (Case 1). The beam velocity is 10 times the thermal speed of the ambient electrons. In this case, strong reflection of the beam electrons has been observed in the one-dimensional simulation (Okuda and $\mathrm{Kan}, 1987)$. A $64\left(\mathrm{~L}_{x}\right) \times 512\left(\mathrm{~L}_{y}\right)$ spatial grid was used for most of the simulation results reported here except for Case 5 where a $128 \times 512 \mathrm{grid}$ is used. The beam radius $\mathrm{L}_{b}=8 \Delta$ where $\Delta$ is the grid size was chosen. There are 4 electrons and ions per grid for the ambient plasma in the simulations. The Debye length of the ambient electrons and ions is chosen equal to $\Delta$. We assume that the amblent magnetic field is reliatively weak so that $w_{p e}=2 \Omega_{e}$ is chosen, which may be realized at low ionospheric altitudes. Here ${ }^{\omega}$ pe and $\Omega_{e}$ are the ambient electron plasma frequency and electron gyrofrequency, defined by $\omega_{p e}=\left(4 \pi n_{o} e^{2} / m_{e}\right)^{1 / 2}$ and $\Omega_{e}=e_{o} / m_{e} c$.

Since the ambient electrons are assumed to be a Maxwellian distribution at $t=0$ given by 


$$
f(\underline{v})=\frac{n_{0}}{(2 \pi) v_{e}^{2}} \exp \left(-\frac{v^{2}}{2 v_{e}^{2}}\right)
$$

the thermal current to the spacecraft across the $y=0$ plane due to the ambient electrons is given by, in the absence of an electric field,

$$
I_{-}=-e \int_{0}^{L_{x}} d x \int_{-\infty}^{0} v_{y} d v_{y} \int_{-\infty}^{\infty} d v_{x} f(\underline{v})=\frac{e n_{0} e^{L} x}{(2 \pi)^{\frac{1}{2}}}
$$

where $v_{e}$ is the ambient electron thermal speed and $e>0$ is the absolute value of the charge of an electron. Similarly, the thermal ion current $\ddot{i}_{+}$is given by

$$
I_{+}=-\frac{\operatorname{env}_{i} L_{K}}{(2 \pi)^{\frac{1}{2}}}
$$

so that $\left|\mathrm{I}_{+} / \mathrm{I}_{-}\right|=\mathrm{v}_{\mathrm{i}} / \mathrm{v}_{\mathrm{e}}=\left(\mathrm{T}_{\mathrm{i}} / \mathrm{T}_{\mathrm{e}}\right)^{1 / 2}\left(\mathrm{~m}_{\mathrm{e}} / \mathrm{m}_{\mathrm{i}}\right)^{1 / 2} \ll 1$. Typically, $\mathrm{T}_{\mathrm{e}} / \mathrm{T}_{\mathrm{i}}=1$ and $m_{e} / m_{i}=1 / 100$ have been chosen for the simulations. In the absence of beam electrons, ths electron and ion fluxes must be equal in order to maintain charge neutrality. As a result, an ambipolar electric field will be generated which then forces the two rluxes to be the same. It is well known that the resultant flow speed is the ion sound velocity. In the presence of beam electrons, particle fluxes given by Eqs. (2) and (3) must be modified since a much stronger electric field is generated in a plasma, as we see later.

The injection current to the ambient plasma from the spacecraft is given by

$$
I_{0}=-e n_{b} v_{o} L_{b}
$$


In this Case $1,\left|I_{-} / I_{0}\right|=\left(v_{e} / v_{0}\right) \cdot\left(L_{x} / L_{b}\right) /(2 \pi)^{\dagger / 2}=0.32$ so that the injection current is much larger than the thermal current. Note, however, the thermal currents defined by Eqs. (2) and (3) are obtained in the absence of an electric field. As we shall see, the injection of beam electrons will generate a large electric field which can significantly modify the return current.

Eigure 2 indicates the instantaneous plots of the been and ambient electrons in $x-y$ space and in phase space at $\omega e^{t}=20$. Beam electrons shown in Fig. 2(a) are observed to expand rapidly across the magnetic field after they are injected isto the ambient plasma. The expanded beam radius is several times the originaI beam radius suggesting that the beam density after expansion is only a fraction of the ambient density. Despite the rapid beam expansion, there is no sign of the reflection of beam electrons back to the spacecraft and the charging of the spacecraft remains negligible, Figure 2(b) indicates the location of the ambient electrons whose initial locations at $t=$ 0 lie along the beam line. While the ambient electrons are uniformly distributed. in the simulation, only those electrons whose initial locations ars along the beam are chosen in order to study the response of the ambient electrons. It is clear that these electrons can be identified as test particles tc study the motion of the ambient electrons across and along the magnetic field. Rarefaction of the censity of the ambient electrons near the spacecraft can be seen clearly, suggesting the loss of the ambient electrons in that region to the spacecraft. This is accomplished by an electric field which accelerates the ambient electrons toward the spacecraft.

Beam-electron phase for space, $y-v_{y}$, shown in Fig. 2(c), shows familiar holes and vortices characteristic of beam-plasma instability seen in onedimensional simulations (Okuda et al., 1987). The phase space, $y-v_{y}$, of the ambienc electrons in Fig. 2(d) shows that a fraction of the ambient electrons 
are accelerated beyond the beam energy as a result of trapping. These observations agree with the results of one-dimensional simulations except that, in one-dimension, beam propagation is possible only when the beam density is substantially smaller than the ambient density (OkLda and Kan, 1987). In two-dimensions, beam electrons spread radially as shown in Fig. 2 (a) which reduces the beam density substantialiy below the ambient density. In this respect, there is good agreement between one- and two-dimensional simulations.

Eigure 3 indicates (a) the electrostatic potential contour, (b) the average electrostatic potential $\Phi(y)=\int \phi(x, y) d x / L_{x},(c)$ the ambient electron velocity distribution along magnetic field, and (d) the beam-electron velocity distribution along magnetic field at $\omega_{\mathrm{pe}} t=20$. Coherent structures are clearly seen in the potential contnurs near the spacecraft. The transverse structure across magnetic field is determined by the radial width of the beam while the longitudinal structure along magnetic field is determined frcm the wavelength associated with the beam-plasma instability given by $\lambda=2 \pi \bar{v} / \omega_{\text {pe' }}$ where $\bar{v}$ is the average bean speed in the plasma. $\bar{v}$ is substantially smailer than the initial injection speed.

The spatially averaged electrostatic potential \$ shown in Fig. 3(b) at ${ }^{\omega} \mathrm{pe}^{t}=20$ decreases slowly toward the spacecraft from $\Phi\left(y=L_{y}\right)=0$ until $y=$ $100 \Delta$, where the potential structure begins to show an oscillatory structure. The potential of the spacecraft at this moment is negative, however, and the spacecraft potential has been observed to fluctuate in time suggesting the spaceerast charging at this beam density is negligible.

Ambient electron velocity distribution shown in Fig. 3(c) indicates a presence of accelerated electrons toward the spacecraft, $v_{y}<0$, as weil as in the direction of beam injection, $v_{y}>0$. It is clear that there are more 
electrons accelerated toward the spacecraft than trapped by the beam-plasma instability. Therefore, the flux carried by the ambient electrons accelerated toward the spacecraft $\left(v_{y}<0\right)$ is larger than that given by $E q$. (2) which assumes a thermal Maxwellian distribution without a net drift.

Beam electron velocity distribution shown in Fig. $3(d)$ indicates a rapid spread in velocity space. High energy electrons beyond the injection energy is seen; however, most of the beam electrons are decelerated via beam-plasma instability.

At a later time, $\omega_{p e} t=50$, we found the beam can propagate more or less freely at this density as shown in Fig. 4. Bearn electrons shown in Fig. 4(a) indicate that they propagate into the ambient plasma without reflection back to the spacecraft. As seen at an early time, radial spread reduces the beam density below the ambient density so that the spaceoraft charging remains small.

Beam phase-space, $y-v_{y}$, shown in Eig. 4(b) indicates the persistence of the beam-plasma instability as in the case of one-dimensional results. Ambient electron flux toward the spacecraft, as well as the high energy tail in the direction of the beam injection is clearly seen in Fig. 4(c), It is interesting to observe that the average electron density $\bar{n}_{\mathrm{e}}(x)=\int \mathrm{n}_{\mathrm{e}}(x, y) d y / L_{y}$ indicates a sharp peak along the beam as shown in Fig. 4(d). While the beam electrons spread radially after injection, the beam electrons attract ambient. ions and at the same time repel the ambient electrons thereby estabiishing an equilibrium density profile. Ion density is very similar to the total electron density shown in Fig. 4(0) which guarantees charge neutrality. The density depletion of the ambient electrons along the beam remains small compared to the ion density bujldup, Becalise of the large ion gyroradii, it is much easier to move ions across the magnetic field than to move 
electrons. In fact, ions may be treated as unmagnetized so that their motion across the magnetic field is primarily controlled by the radial electric Field.

Figure 5 summarizes the total number of particles in the plasma. Figure 5 (a) indicates the total number of beam electrons $\mathrm{N}_{\mathrm{b}}$ in time while $\mathrm{Fig}$. $5(\mathrm{~b})$ gives the total number of ambient electrons $N_{e}$ in the plastia. Here $N_{0}=$ $n_{0} L_{x} L_{y}$. It is clear that the number of the beam electrons increases with time at the injected rate and the ambient electrons are lost at a rate almost equal to the injection rate of the beam electron, leaving a very small net change of electrons $\mathrm{N}_{e}=\mathrm{N}_{b}+\mathrm{N}_{a}$ as shown in Fig. 5(c). The total number of ions remains almost unchanged as shown in Fig. $5(d)$ so that the net charges in the system is negligible.

Figure 6 shows the total current along the magnetic field carried by the beam $I_{b}$ (a) and ambient electrons $I_{a}(b)$ in the plasma normalized by the injection current $I_{0}$ defined by

$$
I_{a, b}=-e \int_{r} v_{y} f_{a, b} d \underline{q} d x d y / I_{o} L_{y},
$$

Which is normalized by the total injection current in the system. We observe that the beam current and the ambient current more or less cancel out each other leaving little net current $I=I_{a}+I_{b}$ as shown in $F i g \cdot 6(c)$. Note the beam current increases at a rate about one-half of the injection rate given by the broken line in Fig. $6(a)$ suggesting the average beam speed in a plasma is $v_{0} / 2$. This agrees with the beam electron velocity distribution shown in Fig. 2 (d) and also explains that the wavelength of the phase-space modulation in Fig. $4(b)$ is about $\pi v_{o} / \omega_{p e}$. The spacecraft potential $\phi_{s}$ shrwn in Fig. $j(d)$ oscillates at a frequency near $\omega_{\text {pe }}$ and does not show any net potential in 
agreement with une-dimensional results at low beam density (Okuda and Kan, 1987).

In the folicwing, we shall study the dependence of the beam propagation on beam density, beam energy, magnetic field strength, and the surface areas of the spacecraft, respectively.

\section{(A) Increasirig the beam dersity (Case 2)}

When the beam density is increased to $n_{b} / n_{0}=2$ whiie keeping all the other parameters the same (Case 2), we found that a significant fraction of beam electrons is reflected back to the spacecraft.

Shown in Fig. 7 are (a) the bear electron spatial distribution in $(x, y)$, (b) the ambient electron spatial distribution in $(x, y),(c)$ the beam electron phase-space, $y-v_{y}$, and (d) the ambient electron phase-space, $y-v_{y}$ at $w_{p e} t=$ 15. It is clearly seen that the oeam electrons are reflected back to the spacecraft after injection. Rapid spread in radius of the beam electrons after injection as in Case 1 is fnllowed by the reflection so that a significant fraction of the beam electrons goes back to the spacecraft. Ambient electron flux is not sufficient to provide charge neutrality so that some beam electrons must return to the spacecraft. This can be seen in Fig. $7(b)$ where the ambient electrons located along the beam line are plotted. Near the spacecraft, the electrons are depleted to the spacecraft and no more electrons are available which can be used to seutralize the spacecraft. Phase-space distributions shown in Figs, $7(\mathrm{c})$ and $7(\mathrm{~d})$ show that most of the beam electrons are reflected near the spacecraft, rather than propagating away freely from the spacecraft so that no slear signs of beam-plasma instabilities are visible. These results are similar to the one-dimensional results at high beem density (Okuda et al., 1987). 
This is also confirmed from the electrostatic potential contours shown in Fig. $8(a)$ and the averaged potential $\delta(y)$, Fig. $8(b)$ at $\omega_{p e}=15$. The potential contours are clustered near the spacecraft and the spacecraft potential stays positive in this example, confirming that the spacecraft is charged positive throughout the entire simulation. The value of the spazecraft potential is $\mathrm{e} \Phi(\mathrm{y}=0) / \mathrm{T}_{e}=50$ so that the potential energy $\mathrm{e} \Phi$ is about one-half of the beam energy.

At a later time, $\omega_{p e} t=50$, beam electrons spread into the ambient plasma at a rate slower than the injection rate. Figures $g(a)$ and $g(b)$ show the locations of the beam electrons in $(x, y)$ and in phase space $\left(y, v_{y}\right)$. It is clear that the majority of the beam electrons are concentrated near the spacecraft. Potential contours shown in Fig. $9(c)$ also confirm that a strong electric field exists only near the spacecraft. Ambient electron velocity distribution along the magnetic field shown in Fig. $9(d)$ indicates the generation of a beam flowing into the spacecraft. Clearly these ambient electrons are accelerated via the positive potential of the spacecraft.

Figure 10 shows the time history of (a) the total number of beam electrons, (b) the ambient electrons, (c) the total electrons and, (d) the ions in the plasma. The total number of the beam electrons increases only at one-half of the injection rate shown by the broken line, suggesting the other one-half of the beam electrons are reflected back to the spacecraft. The ambient electrons are lost to the spacecraft mare or less cancelling the beam flux as shown in Fig. 10(b). The total number of electrons in the plasma shown in.Fig. 10(c) and the total number of ions in Fig. 10(d) show that the number of the electrons is slightly more than the number of the ions, suggesting the spacecraft is charged positive in this case. 
Figures $11(a)$ and $11(b)$ show the current carried by the ambient electrons $I_{a}$ averaged over the plasma region and the spacecraft potential. Again, we note the absolute value of the return current is smaller than the injection current shown by the broken line. The beam current more or less cancels the ambient current so that the net current is found to oscizlate around zero. The spacecraft potential shown in Fig. $1:(b)$ osclllates around the mean positive potential of $e_{s} / T_{e}-40$, which is about $40 \%$ of the injection energy.

We have also run a simulation in which the beam velocity is increased by a factor 2 so that $v_{0} / v_{e}=20$ while keepirig the beam density twice that of the ambient density as in Case 2 . We found that the spacecraft potential remains positive at around $e \phi_{s} / T_{e} \sim 200$ confirming that the spacecraft potential increases with the injection energy, provided the peam density remains sufficiently high relative to the ambient plasma density. This appears to have been observed in the SEPAC data as will be discussed in Sec. IV.

\section{(B) Increasing the beam energy (Case 3)}

We shall now study what happens when the beam energy is increased. We use the same parameters as in Case 1 except for the beam velocity, which is now increased to $v_{0} / v_{e}=20$ (Case 3). The injection current defined by Eq. (4) remains the same as in Case 2 so that it is twice that of Case 1 . This amount of injection current was too large compared with the thermal return current so that about one-half of the beam electrons is reflected back to the spacecraft in Case 2.

As we shall see, the results of the present case are different from those in Case 2 despite the fact that the injection currents are the same for both cases. Instead, we find that the beam electrons manage to propagate into the plasma without reflection, similar to the first case. The results of the 
simulations at $w_{p e} t=25$ are shown in Fig. 12 for, (a) the locations of the beam electrons, (b) the ambient electron phase space, $y-v_{y}$, (c) the potential contours and, (d) the averaged potential $\$(y)$. It is clear from Fig. 12(a) that the beam electrons can propagate into the ambient plasma wich little reflection so that the beam-plasma instabiity develops as seen in the phase space of the ambient electrons in Fig. 12(b) where trapped paricicle vortices are clearly seen. The electrostatic potential contours show a presence of regular patterss determined by the size of the beam radius and the wavelength of the beam-plasma instability as seen in Fig. 12(c).

The spacecraft charged up positively at this moment as seen in Fig. 12(d) with a wavy structure away from the spacecraf't characteristic to the beamplasma instability. These results of simulation are similar to what we have seen in Case 1 rather than the results found in Case 2. These results are in agreement with the one-dimensional results where the injection surrent much larger than the thermal return current can he injected only when the beam velocity is increased while keeping the beam density low lokuda et al., 1987). In this Case 3 , the injection current $I_{0}$ is 6.25 times the thermal return current, $\left|I_{0} / I_{-}\right|=6.25$. Beam electron velocity distribution along the magnetic field indicates the presence of a beam toward the spacecraft similar to that found in Case 1. These electrons represent an enhanced return flow of the ambient electrons.

The total number of beam electrons in a plasma increases Iinearly in time at the injection rate as shown in Fig. 13(a) while the number of ambient particles decreases in response to the injected electrons as shown in Fig. 13(b). The total number of electrons stays about the same as shown in Fig. 13(c) while the net charge of the system $\delta N=N_{i}-N_{e}$ oscillates at a frequency close to the electron plasma oscillations as shown in Fig. 13(d). 
The beam current in the plasma increases with time at a rate of one-half of the injection current as shown in Fig. 14(a) which is nearly cancelled by the return flow of the ambient electrons shown in $\mathrm{Fig} .14(\mathrm{~b})$. The net current oscillates around zero mean with the frequency close to the electron plasma frequency. The time history of the spacecraft potential indicates an oscillation around zero mean in sharp contrast to Case 2 where the spacecraft is found to charge up positively, although the infection current remains the same for Case 2 and Case 3.

\section{(C) Increasing the magnetic field (Case 4)}

When the ambient magnetic field is increased to $\Omega_{e} / \omega_{\text {pe }}=2$ from $\Omega_{e} / \omega_{p e}=$ 0.5 in Case 1 , we may expect tre electron motion will become one-dimensional since the motion across the magnetic field becomes prohibited. This does not mean, however, the whole dynamies can be approximated by a one-dimensional model since the width of the electron beam remains small in comparison with the spacecraft or the propagation length in the direction of magnetic field. Electric field lines can bulge out of the beam electrons in two-dimensions which weakens the electric field along the magnetic field. As a result, the reflection of the beam electrons is reduced in two-dimensions compared with the one-dimensional simulations. This makes beam propagation possible even though the radial expansion of the beam is much smaller compared with Case 1 when the magnetic field is weak.

Using the same parameters as in Case $t$, the ambient magnetic field is increased to $\Omega_{e} i \omega_{p e}=2$ which may be relevant to the ionospheric plasma at an altitude of a few hundred $\mathrm{km}$. The results shown in Fig. 15 are for (a) the beam electron spatial distribution in the $(x-y)$ plane, (b) the ambient electron spatial distribution, (c) the beam electron phase-space, $y-v y$, and 
(d) the ambient electron phase space, $y-v_{y}$, at $\omega_{p e} t=50$. Compared with Figs. $2,4,7,9$, and 12 which correspond to a weaker magnetic field, it is olear that the radial expansion of the beam and the ambient electrons are much smaller in tine present rase. The beam electrons, in fact, propagate along the magnetic field without appreciable radial expansion as shown in Fig. 15(a). The ambient electrons shown in Fig. 15(b) also indicate the absence of any significant radial diffusion and the density is depleted near the spacecraft. In Figs. 15(c) and 15(d) vortices and holes in the phase space are clearly seen both for the beam and the ambient electrons, indicating the presence of beam plasma instability.

The electrostatic potential contours shown in Fig. $16(3)$ confirm that the structure of the electric field is similar to that in Case 1 so that the electrostatic reflection of the beam electrons back to the spacecraft is not much stronger here. The ambient electron velocity distribution along the magnetic field shown in Fig. $\uparrow 6(b)$ is again similar to Fig. $4(0)$ indicating a presence of the enhanced return flux to the spacecraft and the accelerated electrons in the direction of beam propagation. It is interesting to observe that the ion density becomes peaked at the center of the system as shown in Fig. 16(a). This is because the electrons cannot diffuse across the magnetic field in two-dimensional simulation where the $\mathrm{cE} \times \underset{\sim}{\mathrm{g} / \mathrm{B}^{2}} \mathrm{drift}$ is in the $z$-direction (which is the ignorable coordinate). Ions are attracted by the electrons since the ion mobility across the magnetic field is much larger. The total electron density is almost identical to the ion density shown in Fig. 16(a). Beam electrons spread ut more along the magnetic field as shown in Fig.16(d) where the energy of the accelerated electrons exceed the beam infection energy significantly. This is similar to the earlier onedimensional results where the high energy electrons can attain an energy twice that of the injection energy (Okuda et al., 1985). 
Eigure 17 summarizes the total number of the electrons and the current of the system. The total number of beam electrons increases monotonically without reflection which accompanies the decrease of the eoual number of the ambient electrons as shown in Figs. $17(a)$ and $17(b)$. These results are sinilar to Figs. $7(a)$ and $7(b)$. The current carried by the beam electrons shown in Fig. 15(c) is about one-third of the injection current shown by the broken line. This is more or less cancelled by the current carried by the ambient electrons flowing into the spacecraft shown in Fig. 17(d) so that no net charging takes place.

\section{(D) Increasing the spacecraft surface (Case 5)}

Finally, we shall study what happens when the surface area of the spacecraft, $L_{x}$, is increased relative to the beam dimension, $L_{b}$. According to Eqs. (2) and (4), the return current carried by the ambient electrons is proportional to the surface area so that if the area is increased relative to the beam radius, it should be possible to inject a beam with higher density without significant reflection. If the bean density is too high compared with the ambient density, on the other hand, the local electric field may be strong enough to reflect the beam electrons back to the spacecraft as in the case of one-dimensional simulations (Okuda and Kan, 1987).

In Case 5 , the simulation system is increased in $\mathrm{x}$-direction by a factor of 2 from the other cases so that $L_{x}=128 \mathrm{~A}$. He shall assume the beam density is twice the ambient density and the beam injection speed $v_{0} / v_{e}=10$ so that the total injection eurrent is the same as that of case 2 where strong reflection of the injected beam to the spacecraft was found. According to Eq. (2), the return current must increase by a factor of 2 in this case, so that a smaller amount of charging of the spacecraft may be expected to take place. 
Results of the simulations in this case are shown in Fig. 18 for (a) the locations of the beam electrons in $(x-y)$ space, (b) beam-electron phase space in $\left(y-v_{y}\right)$ space, (c) electrostatic potential contour, and (d) the spacecraft potential e $\phi_{s} / T_{e}$ at $\omega_{p e} t=50$. Beam electrons propagate into the ambient plasma without significant reflection as shown in Fig. 18(a) and the phase space indicates a presence of trapped particles in Fig.18(b). Note the rapid radial expansion of the beam electrons near the injection point. Electrostatic potential contours also indicate a coherent structure associated with the beam propagation, and the spacecraft potential, $e_{s} / T_{e}$, oscillates in time, although its mean value is positive, $\mathrm{e}_{\mathrm{s}} / \mathrm{T}_{\mathrm{e}} \sim 25$, suggesting that the spacecraft is charged up to the potential equal to one-quarter of the beam energy .

Time history of the total number of beam and ambient electrons and their currents are shown in Fig. 19. The number of the beam electrons increases linearly in time suggesting no reflection as shown in Fig. 19(a) until the end of the simulation when a small fraction of beam electrons is reflected. The ambient electrons decrease at the injection rate as shown in Fig. 19(b). The beam current, in the plasna increases at a rate roughly one-third of the injection current, which is more or less cancelled oy the return current carried by the ambient electrons to the spacecraft as shown in Fig. 19(c) and (d). The results of this simulation confirm that the increase of the conducting area of the spacecraft helps increase the injection current by drawing more return current to the spacecraft from the surrounding plasma.

\section{D1scussion}

The results of the two-dimensional simulations of the injection of an electron beam into a plasma have been presented in which the beam electron 
density is comparable to or larger than the ambient electron density and the injection current is larger than the thermal return current carried by the ambient electrons. In contrast to the earlier results in one-dimensional simulations, an electron beam can propagate into the ambient plasma when the density is comparable to or larger than the ambient density. This propagation is attained partly because of the radial spread of the beam electrons after injection due to the radial electric field and partly due to the finite radius of the beam which causes the bulging of the electric field along the beam injection.

Spacecraft charging remains negligible even when the injection current exceeds the thermal return current so long as the beam density is not much larger than the ambient density. This result agrees with the one-dimensional simulations where the electric field generated as a result of beam injection accelerates the ambient electrons toward the spacecraft, thereby canceling the injection current. The resultant spacecraft charging remains small.

In order to increase the injection current, it is more effective to increase the beam energy rather than to increase the beam density (Cases 2 and 3). This is consistent with the results found in one-dimensional simulations. When the size of the spacecraft is increased relative to the size of the beam, we found that the bearn current can be increased while no reflection of the ambient electrons takes place (Case 5). This is in agreement with Eq. (2) in which the total return current increases with the conducting area of the spacecraft. The beam surrent in a plasma is generaliy smaller than the injection current for all cases. High energy electrons are found in bith beam and ambient phase-space which contributes to the enhancement of the classical mean-free patin as in one-dimensional results (Okuda et al., 1985). 
The results of the present two-dimensional simulations may be used to analyze data of space experiments with particle accelerators (SEPAC). The SEPAC experiments involved injection of an electron beam into the ionosphere using an electron beam accelerator (Obayashi et al., 1984). It was found that the spacecraft potential increased with the injection current reaching the accelerator voltage of $7.5 \mathrm{keV}$ for a beam ourrent of $100 \mathrm{~mA}$. Psyond that point, the voltage stayed constant, suggesting the beam electrons were not able to leave the spacecraft.

The ambient ionospheric electron density near the $200 \mathrm{~km}$ altitude is $\mathrm{n}_{0} \leq$ $5 \times 10^{5} \mathrm{~cm}^{-3}$ and the temperature $\mathrm{T}=2000^{\circ} \mathrm{K}$ so that the maximum thermal return current would be

$$
I_{-}=\frac{e n_{0} S v_{e}}{(2 \pi)^{\frac{1}{2}}} \leq 50 \mathrm{~mA} \text {, }
$$

where $S=60 \mathrm{~m}^{2}$ is the conducting area on Spacelab 1. Therefore, we find the beam injection currents of $100 \mathrm{~mA}$ and beyond are significantly larger than the maximum thermal return current so that significant charging should take place. This is in good agreement with the results of numerical simulation, suggesting the spacecrartt potential must approach the accelerator potential. The beam density is found from

$$
n_{b}=I_{0} / e v_{0} a
$$

where $a$ is the beam cross section. $a=12 \mathrm{~cm}^{2}$ at the end of the accelerator but it becomes larger as the beam electrons move out of the spacecraft. Assuming a $\approx 200 \mathrm{~cm}^{2}$ at the injection point, 


$$
\mathrm{n}_{\mathrm{b}}=10^{6} \mathrm{~cm}^{-3},
$$

which is about twice the ambient ionospheric density corresponding to Case 2 where a significant charging of the spacecraft and bean reflection take place. Realizing that the electron plasma frequency is $\mathrm{f}_{\mathrm{pe}} \approx 6 \times 10^{6} \mathrm{~cm}^{-1} \ll$ $f_{c e}$, the beam electrons should be able to expand radially once injected into the ionosphere as seen in our simulations for a weak magnetic field.

We should emphasize that while the simulation results reported here are, in ganeral, consistent with the data from SEPAC, the ionosphere is far more complex than the simulation model used here. Eor example, the ionosphere is a weakly ionized plasma so that the neutral gas density is much larger than the plasma density. Even a small amount of additional ionization of the neutral. gas due to high energy electrons can modify the ambient density, thereby modifying the spacecraft potential.

While we have studied only the injection of the electron beam along the ambient idagnetic field, it is important to study the oblique injection since the injection angle in real space experiments cannot be controlled as the spacecraft moves across the magnetic field. Furthermore, at a higher altitude of the ionosphere, the effect of the ambient magnetic field becomes important since the beam electrons cannot expand freely as seen in Case 4 . In such a case beam rotation around the ambient magnetic field and the associated plasma instability is important in deteraining the propagation and the stability of the beam. Such a process can be studied by using a three-dimensional simulation model with elongated grid (Cheng and Okuda, 1977, Okuda et al., 1979). These results will be reported elsewhere. 


\section{Acknowledgments}

The authors are grateful for discussions with Professors N. Kawashima and S. Sasaki and for their information on the SEPAC experiment. This work was supported by National Science Fo'lndation Grant ATM-85-12512 and the United States Department of Energy Contract NO. DE-ACO2-76-CHO-3073. 
References

Artificial Particle Beams in Space Plasma Studies, edited by Bjorn Grandal, Plenum Press, New York 1982.

Cheng, C.z., and H, Okuda, New three-dimensional simulation models for cylindrical and toroidal plasmas, J. Comput. Phys., 25, 133, 1977.

Kawashima, Nobuki, Experimental studies of the neutralization of a charged vehicle in space plasma studies, in Artificial Particle Beams in Space Plasma Studies, edited by Bjorn Grandal, p. 597-626, Plenum Press, New York, 1982.

Obayashi, T., N. Kawashima, K. Kuriki, M. Nagatomo, K. Ninimiya, S. Sasaki, A. Ushirokawa, I. Kudo, M. EJiri, W.T. Roberts, R. Chappell, J. Burch and P. Banks, Space Experiments with Particle Accelerators (SEPAC), in Artificial Carticle Beams in Space Plasma Studies, edited by Bjorn Grandal, p. 659-671, Plenum Press, New York, 1982.

Obayashi, Tatsuzo, N. Kawashima, K. Kuriki, M. Nagatomo, K. Ninomiya, S. Sasaki, M. Yanagisawa, I. Kudo, M. Ejiri, H.T. Roberts, C.R. Chappel, P.L. Reasoner, J.L. Burch, W.L. Taylor, P.M. Eanks, P.R. Williamson, and O.K. Garriott, Science, 225, 195, 1984.

Okuda, H., R. Horton, M. Ono, and K.-L. Wong, Effects of beam-plasma instability on current drive via injection of an electron beam into a torus, Phys. Fluids 28, 3365, 1985.

Okuda, H., R. Horton, M. Ono, and M. Ashour-Abdalla, Propagation of a nonrelativistic electron beam in a plasma in a magnetio field, Phys. Fluids 30, $200,1987$.

Okuda, H., Propagation of an electron beam and spacecraft charging, in Proceedings of the Sixth International Conference on High Powered Particle Beams, Kobe, Japan, 1986. 
Okuda, H., and J.R. Kan, Injection of an electron beam into a plasma and spacecraft charging, Phys. Eluids 30, 209, 1987.

Ckuda, H.; W.W. Lee, and C.Z. Cheng, Electrostatic and magnetostatic particle simulation models in three dimensions, Comput. Phys. Comun. 17, 233, 1979. 
Figure Captions

Fig. 1 A sketch of the two-dimensional simulation model. An electron be am is injected into a fully ionized ambient plasma from a spacecraft located at $\mathrm{y} \leq \mathrm{0}$ and $0 \leq \mathrm{x} \leq \mathrm{L}_{\mathrm{x}}$. The electron beam dimension $\mathrm{L}_{\mathrm{b}}$ is injected along a uniform anbient magnetic field immersed in a uniform ambient plasma whose dimension is $\mathrm{L}_{x} \times \mathrm{L}_{\mathrm{y}}$.

Eig. 2. Results of the simulation at $\omega_{p e} t=20$. (a) The location of the beam electrons in $x-y$ plane, (b) the location of the selected ambient electrons in $x-y$ plane, $\langle c\rangle$ the $y-v_{y}$ phase space for the beam electrins, and (d) the $y-v_{y}$ phase space for the ambient electrons. $64(x) \times 512(y)$ grid, $\omega_{p e}=2 . z_{e}, v_{c} / v_{e}=10, n_{b}=n_{0}$, and $L_{b}=8 \Delta$. Case 1.

Fig. 3. Results of the simulation at $w_{p e} t=20$. (a) Electrostatic potential contour in $x, y$ plane, (b) the average potential distribution $\Phi(y)$, (c) the ambient electron velocity distribution along magnetic field, $f^{a}\left(v_{y}\right)$, and (d) the beam electron velocity distribution along magnetic field, $f^{b}\left(v_{y}\right)$. Case 1 .

Eig. 4. Results of the simulation at $\omega_{p e} t=50$. (a) The locations of the beam electrons in $x-y$ plane, (b) the phase space of the beam electrons along magnetic field, $y-v_{y}$ plane, (c) the velocity distribution of the ambient electrons along magnetic field, $f^{a}\left(v_{y}\right)$, and (d) the total electron density across magnetic field $\vec{n}_{e}$. Case 1. 
Fig. 5. Number of the electrons and the ions in the plasma. (a) the total number of the beam electrons in the plasma, (b) the total number of the ambient electrons in the plasma, (c) the total number of electrons in the plasma, and (d) the total number of ions in the plasma. Case 1.

Fig. 6. Beam and ambient electron currents in the plasma and the spaceoraft potential. (a) The beam current, (b) the ambient current, (c) the net current, and (d) the spacecraft potential $e_{s} / T_{e}$. The injection current in the plasma is shown by the broken line in Fig. 6(a). Case 1.

Fig. 7. Results of the simulation with higher beam density at $\omega_{p e} t=15$. (a) The locations of the beam electrons $x-y$ plane, (b) the locations of the ambient electrons in $x-y$ plane, (c) the beam electron phasespace in $y-v_{y}$, and (d) the ambient electron phase-space in $y-v_{y}$. Case 2 .

Fig. 8. Results of the simulation at $\omega_{p e} t=15$. (a) The electrostatic potential contour and (b) the average potential $\bar{\phi}(y)$ along magnetic field. Case 2.

Fig. 9. Results of the simulation at $\omega_{p e} t=50$. (a) The locations of the beam electrons in $x-y$ plane, (b) the beam electron phase space in $y$ : $v_{y}$ plane, (c) the electrostatic potential contour, and (d) the ambient electron velocity distribution along magnetic field, $f^{a}\left(v_{y}\right)$. Case 2 . 
Eig. 10. The total number of electrons and ions in the system. (a) The number of the beam electrons in the plasma, (b) the number of the ambient electrons in the plasma, (c) the total number of electrans, and (d) the total number of ions. The total number of injected electrons is shown by the broken line in Fig. 10(a).

Fig. 11. Results of the simulation showing the return current due to the ambient electrons (a), and the spacecraft potential (b). The injection current is shown by the broken line in Eig. 11(a). Case 2.

Fig. 12. Results of the simulation with more energetic beam electrons at wpet = 25. (a) The locations of the beam electrons in $(x-y)$ space, (b) the phase-space plot for the ambient electrons, (c) the electrostatic potential coutour, and (d) the average potential distribution along magnetic field. Case 3 .

Fig. 13. Number of the electrons and the net charge at $\omega_{p e} t=25$. (a) The total number of the beam electrons, (b) the total number of the ambient electrons, (c) total number of electrons, and (d) the net charge $\delta N=N_{i}-N_{e}$. Case 3 .

Fig. 14. Currents in the plasma. (a) The beam current and (b) the return current carried by the ambient electrons. The infection current is shown by tio broken line in Fig. 14(a). Case 3. 
Fig. 15. Results of the simulation with a stronger magnetic field at wpe $=$ 50. (a) The locations of the beam electrons in $x-y$ space, (b) the locations of the ambient electrons in $x-y$ space, (c) the beam electron phase space in $y-v_{y}$, and (d) the ambient electron phase space in $y-v_{y}$. Case 4 .

Fig. 16. Results of simulation at $\omega_{p e} t=50$. (a) The electrostatic potential contour, (b) the ambient electron velocity distribution along magnetic field, (c) the average ion density across magnetic field, and (d) the beam electron velocity distribution along magnetic field. Case 4.

Fig. 17. The total number of electrons and the currents in the plastha. (a) The total number of the beam electrons in the plasma, (b) the total number of the ambient electrons, (c) the beam eurrent in the plasma, and (d) the return current carried by the ambient electrons. The infection current is shown by the broken line in Fig. 17(c). Case 4.

Fig. 18. Results of the simulation with a larger spacecraft surface area at $w_{p e} t=50$. (a) The location of the beam electrons in $x-y$ space, (b) the beam phase-space in $y-v_{y}$ space, (c) the electrostatic potential contour, and (d) the time history of the spacecraf's potential, $\mathrm{e}_{\mathrm{s}} / \mathrm{T}_{\mathrm{e}}$. Case 5 . 
Fig. 19. Number of the electrons and the current in the plasma. (a) The total number of the beam electrons in the plasma, (b) the total number of the ambient electrons, (c) the beam current in the plasma, and (d) the return current carried by the ambient electrons. The injection current is shown by the broken line in Fig. 19(c). Case 5 . 


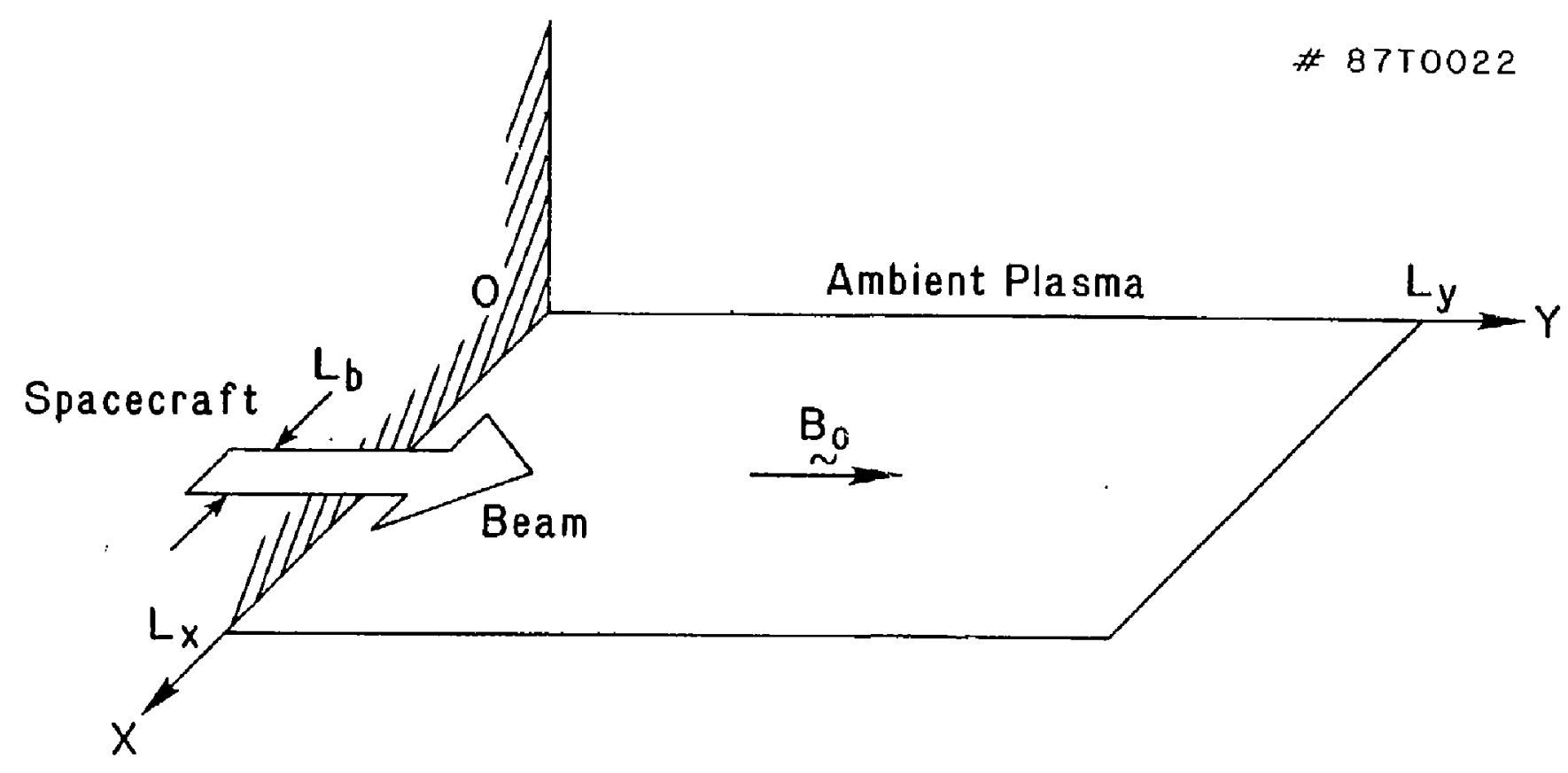



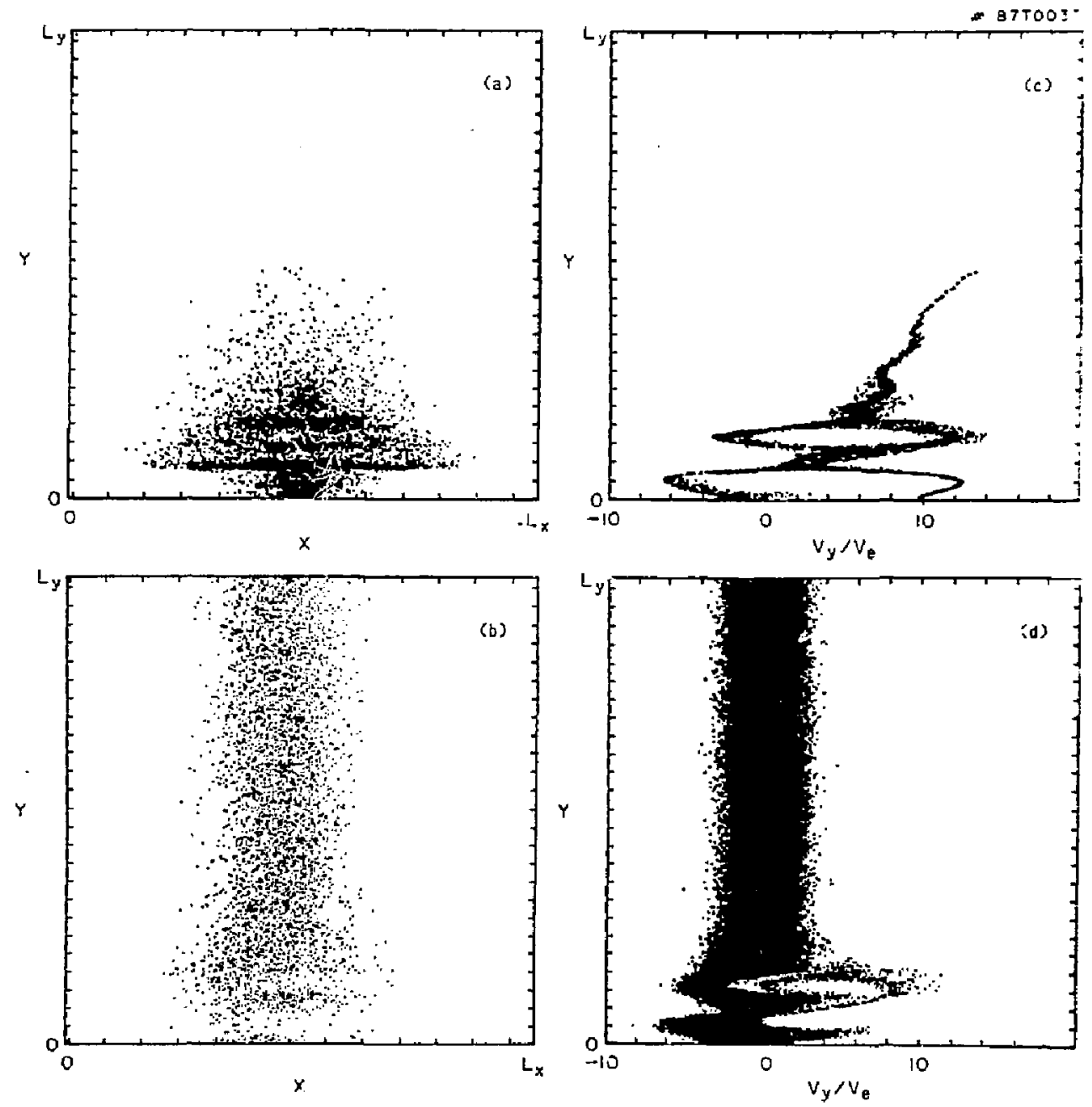

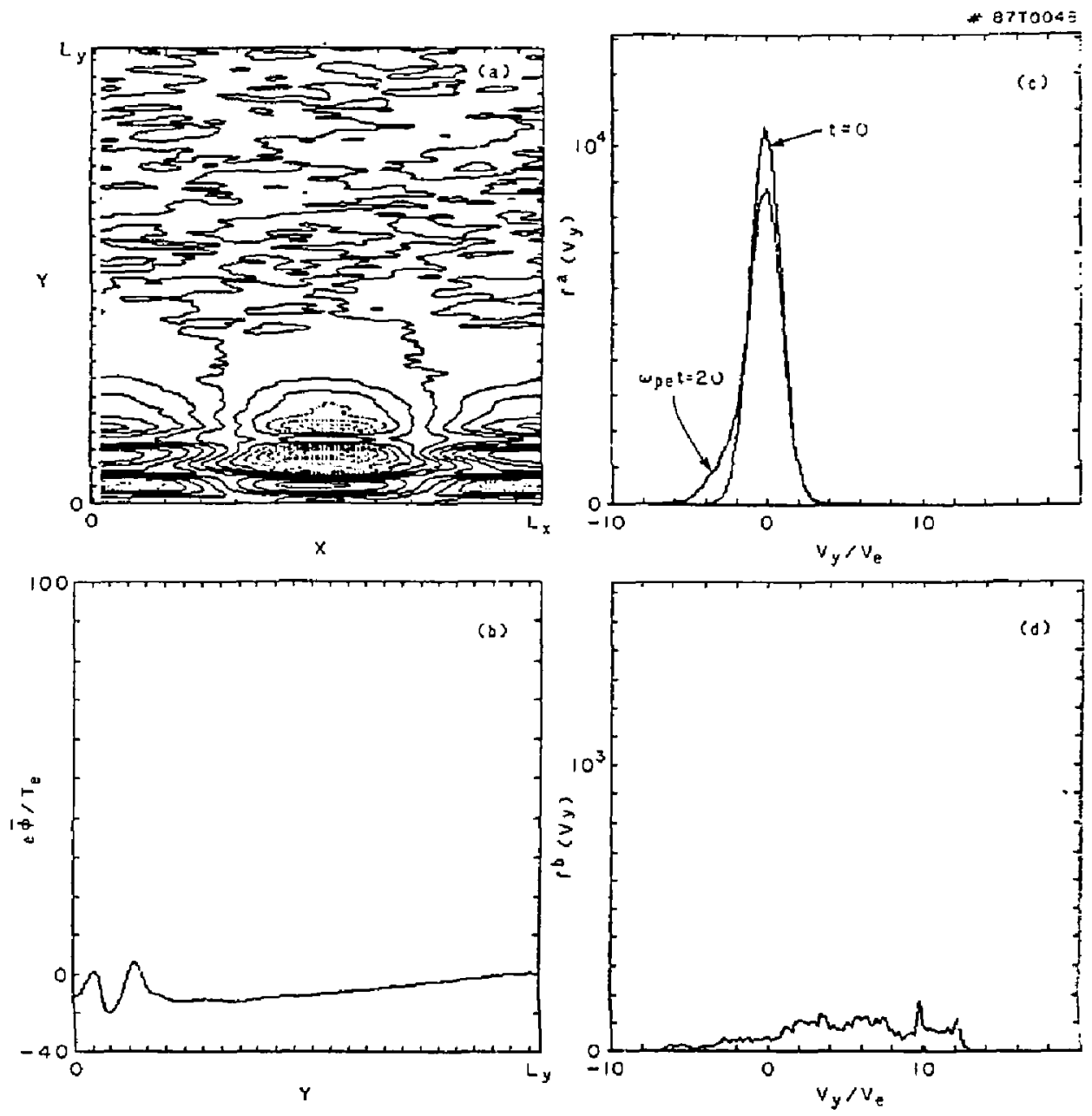

Fig, 3 

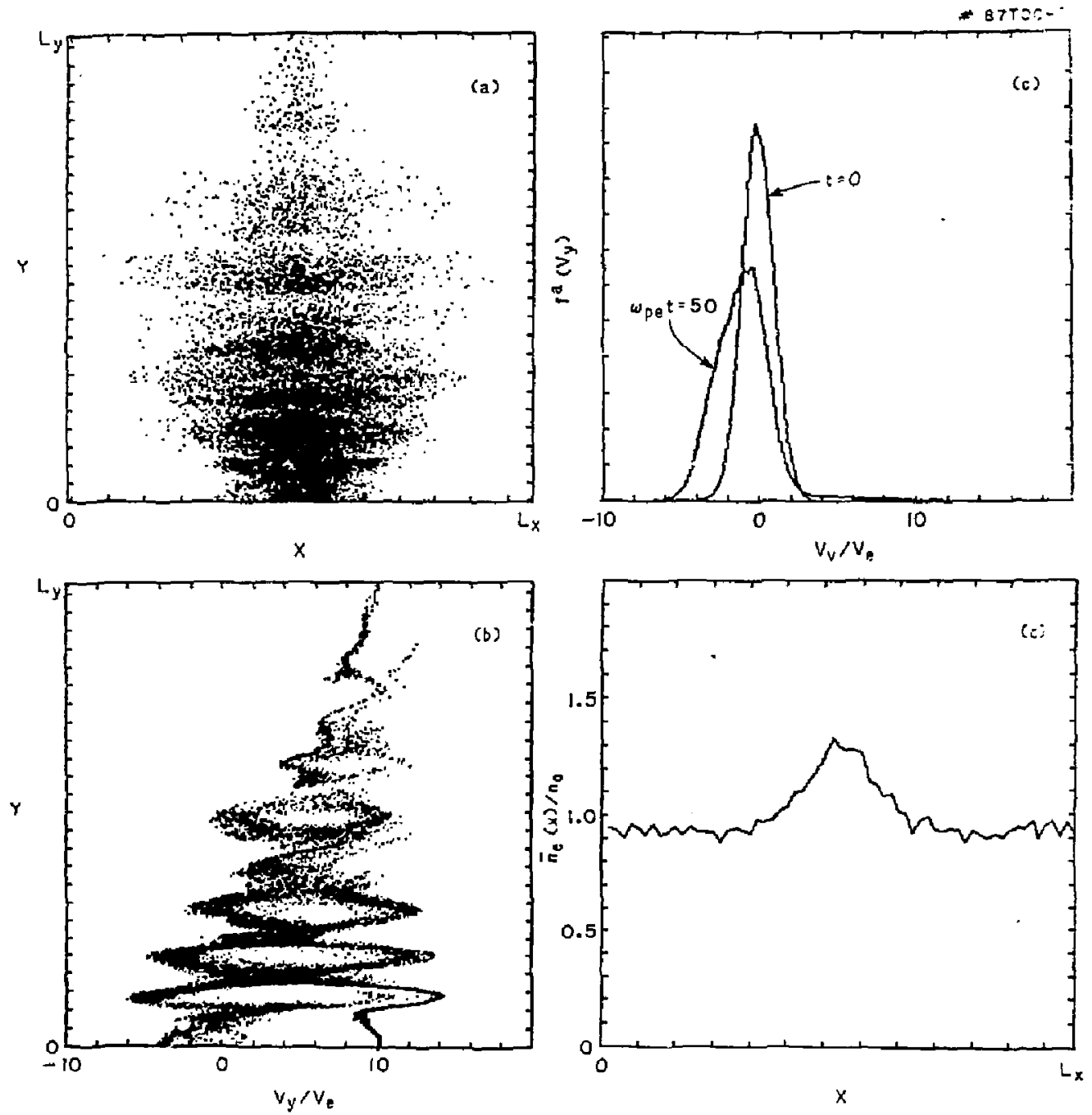

Fig. 4 

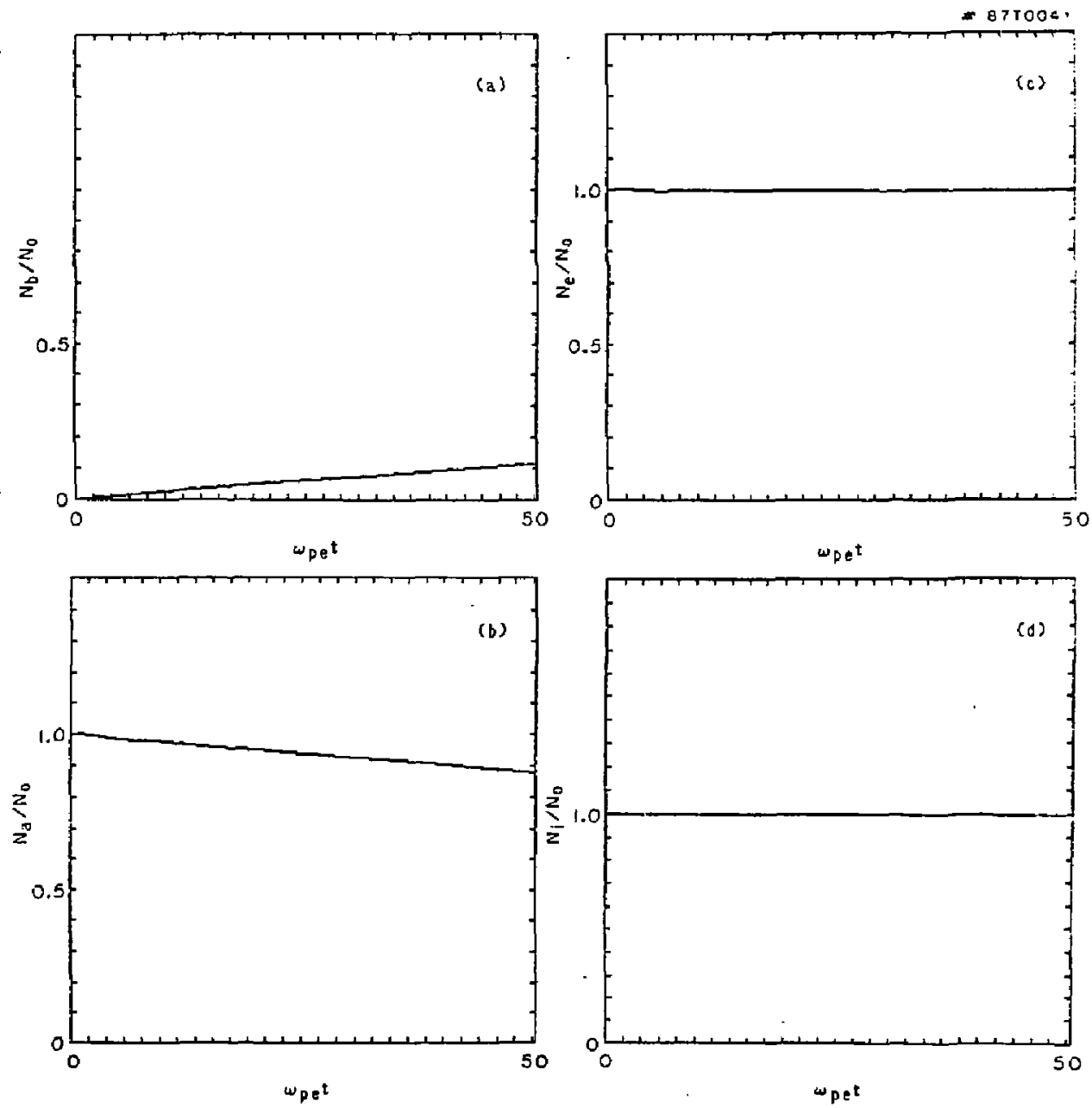

Fig. 5 

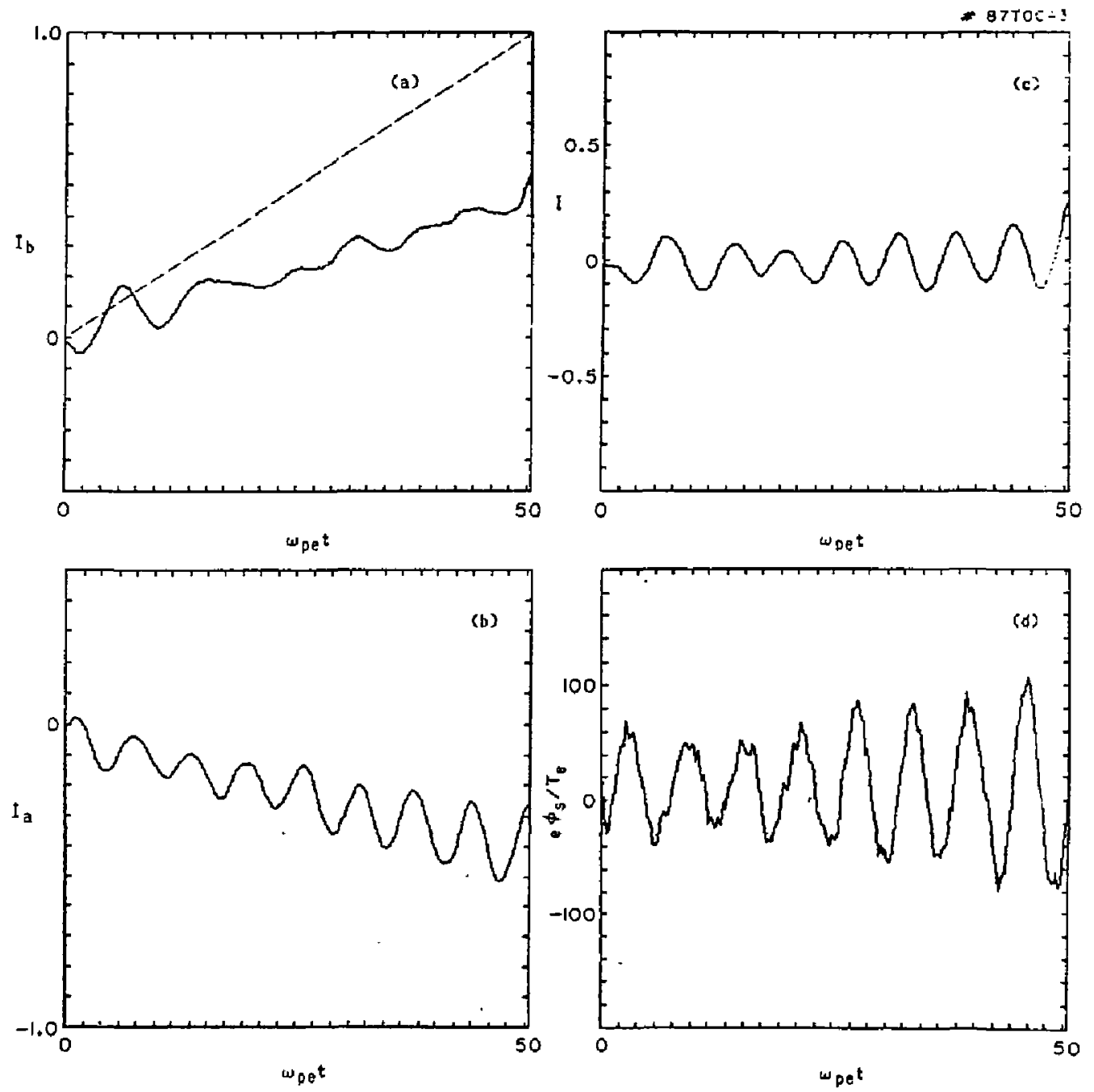

Fig. 6 

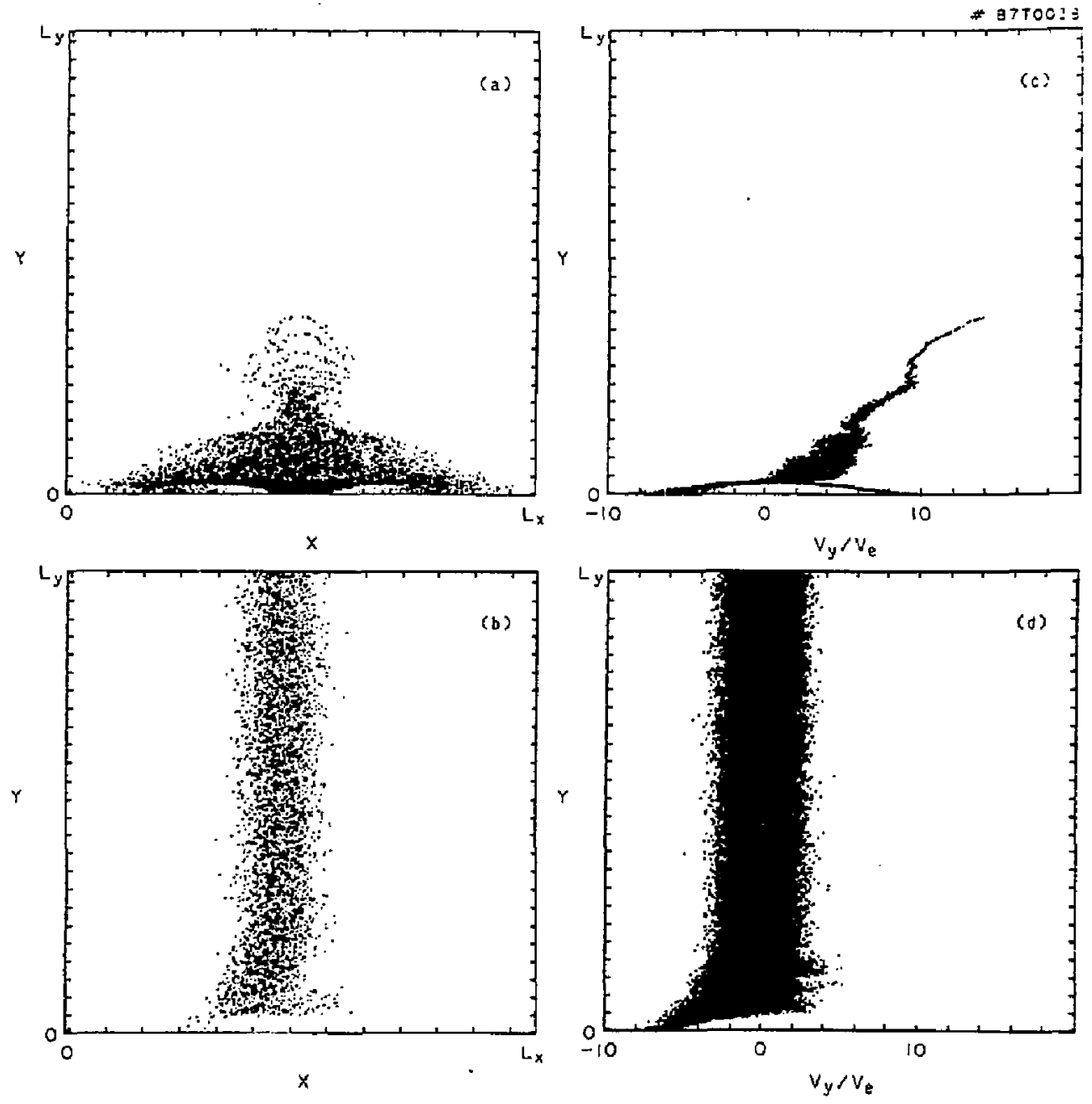

Fig. 7 


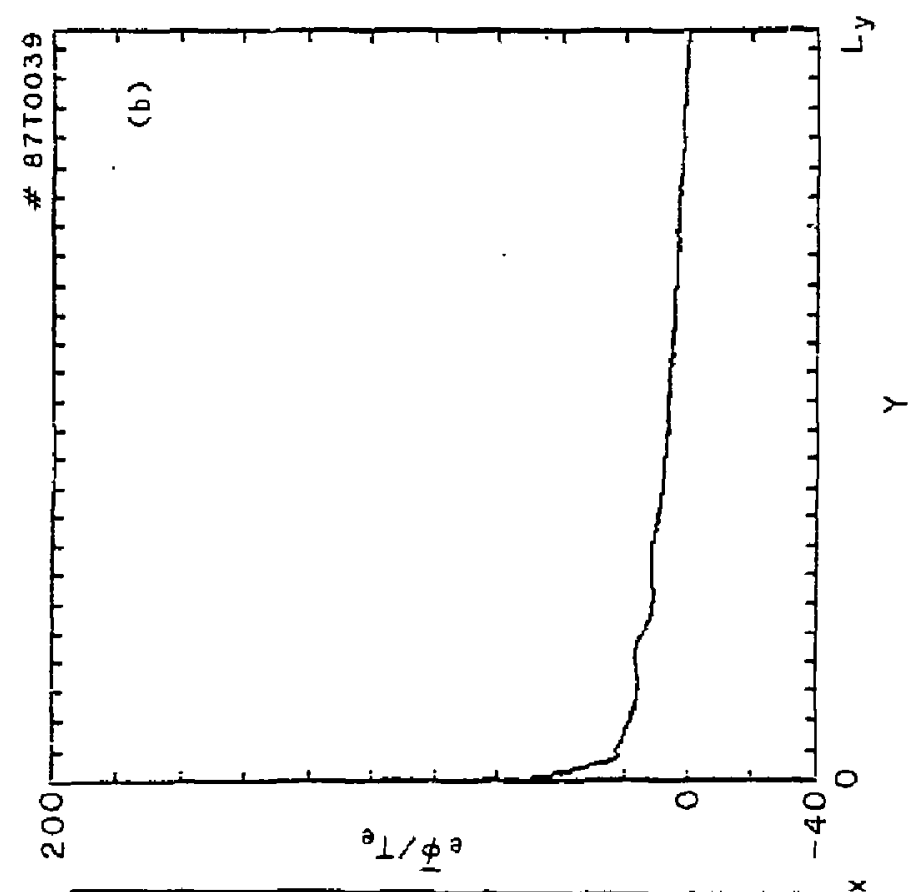

$\underset{\substack{\infty \\ \dot{4}}}{\infty}$

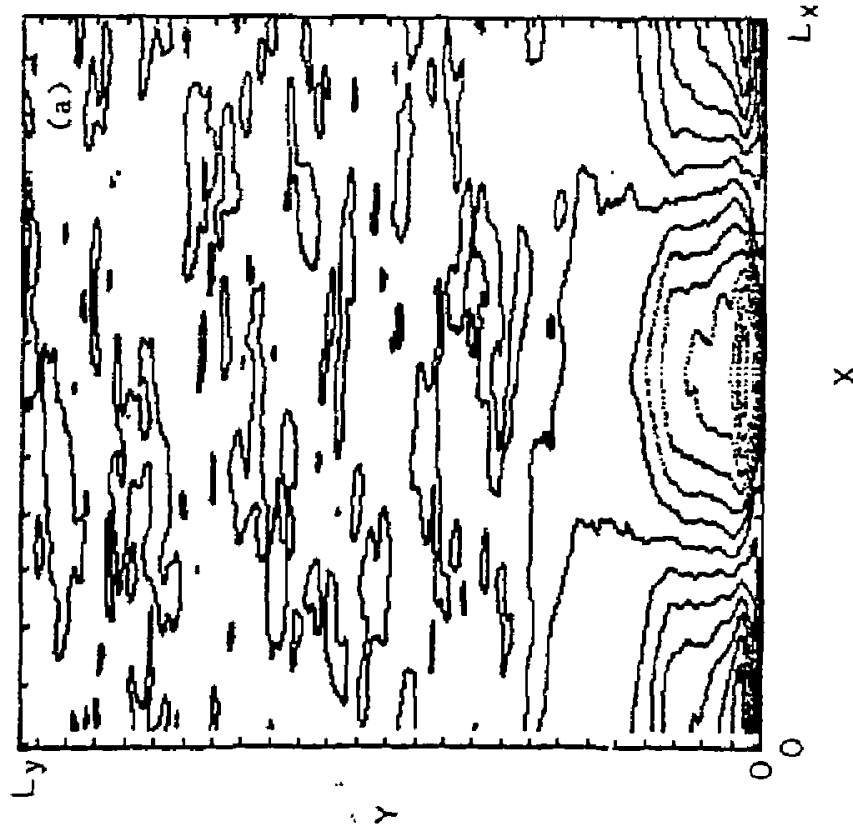



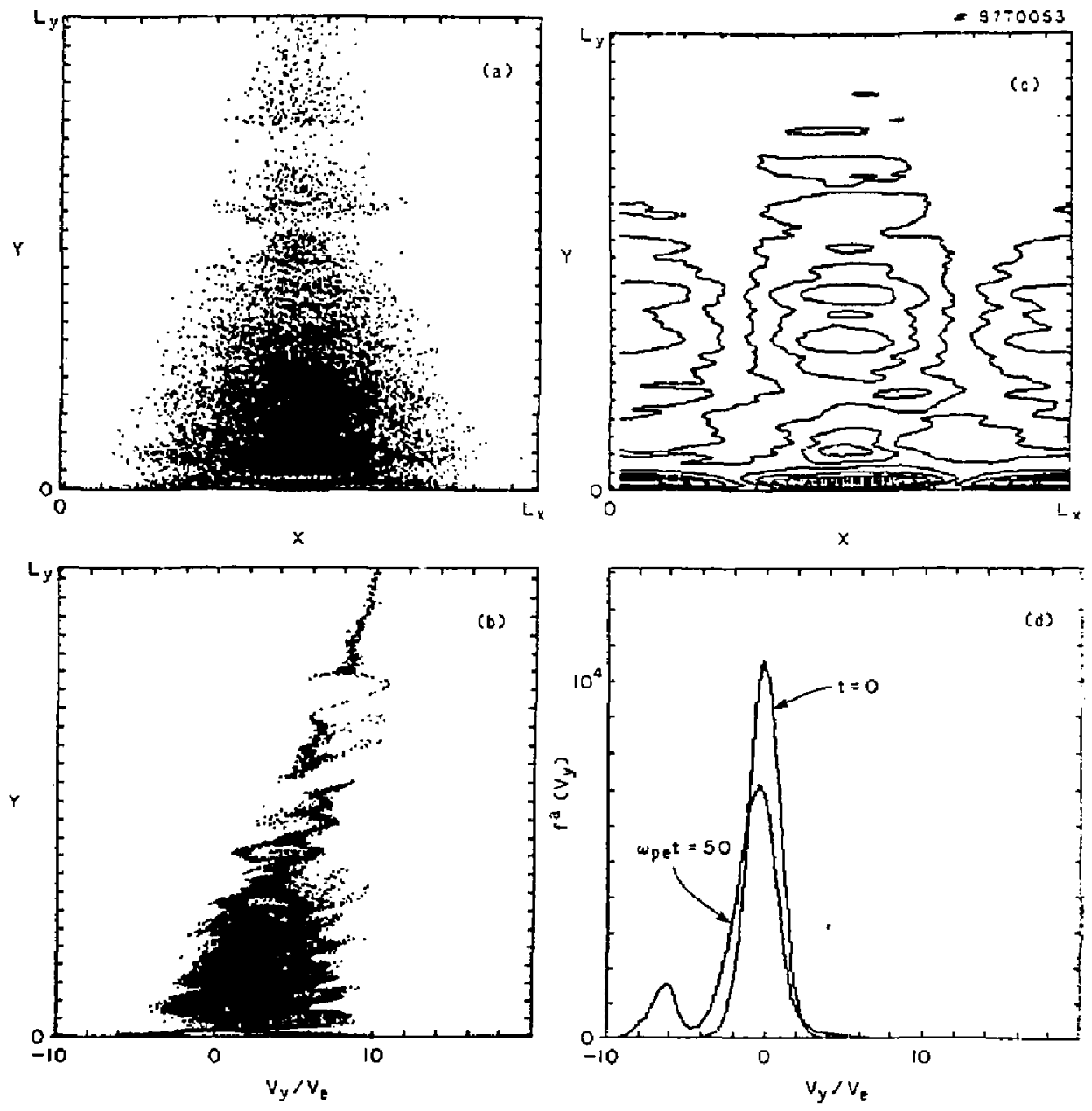

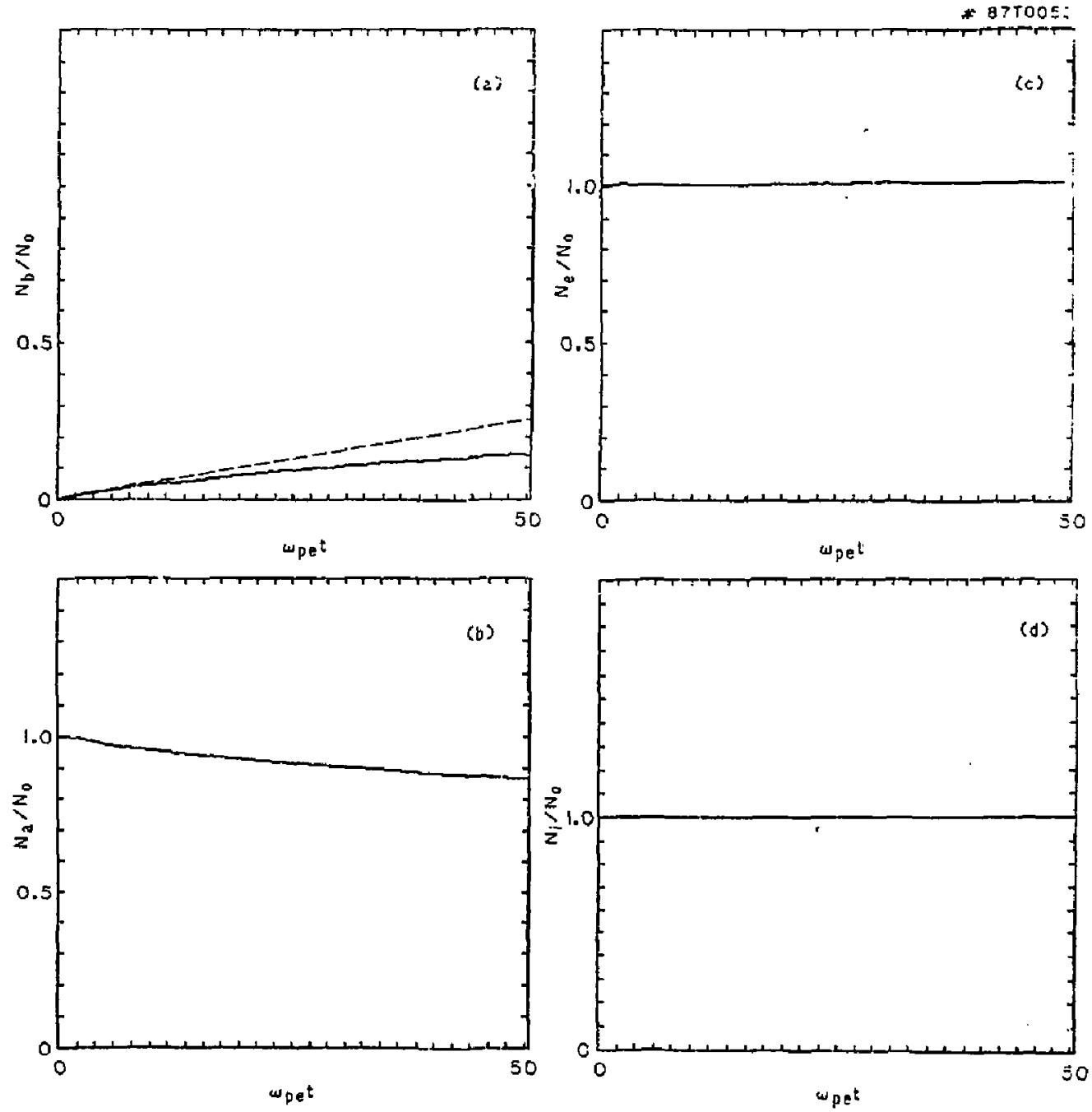

Fig. 10 

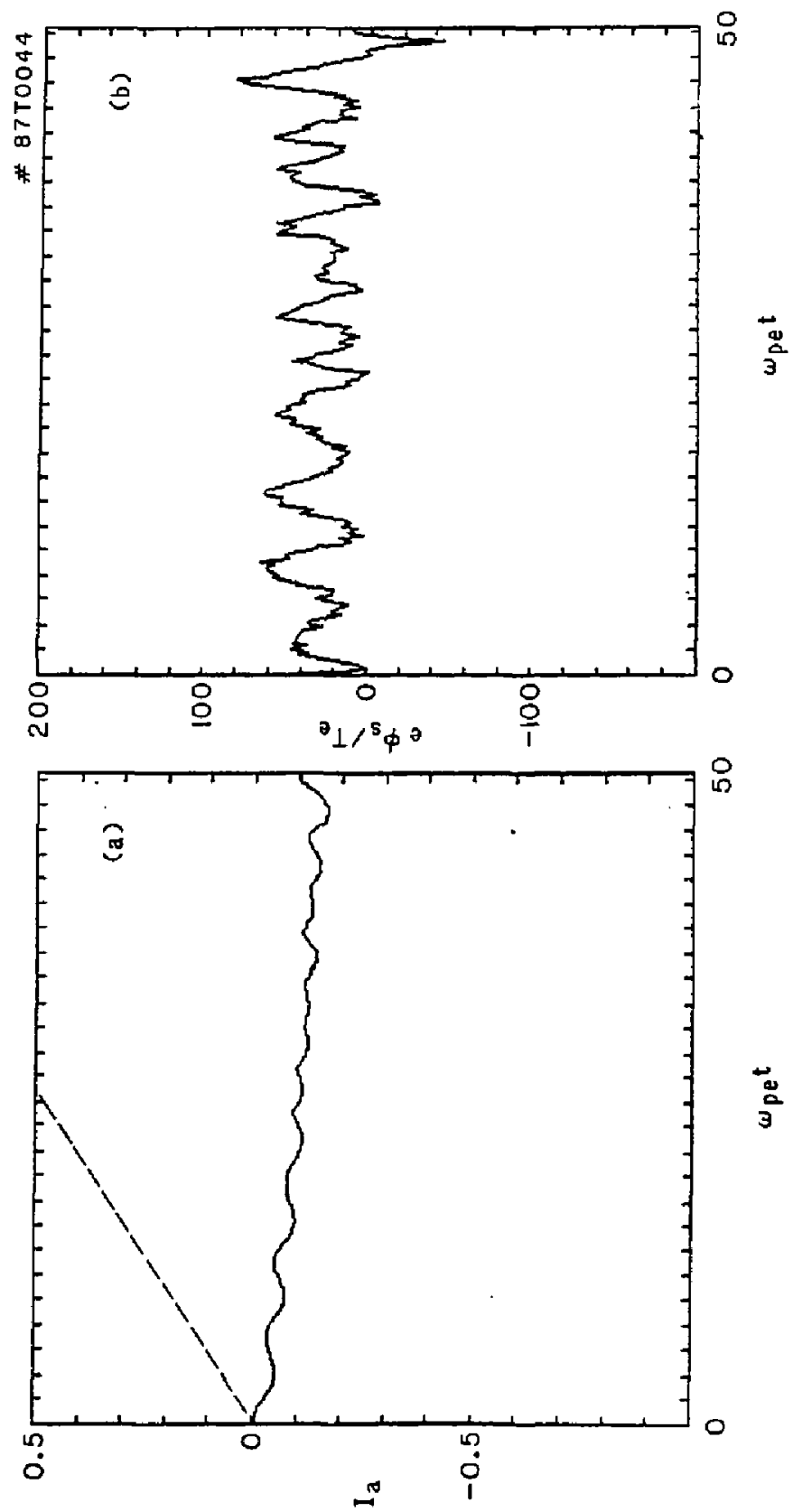

$ت$
-1
0
0 

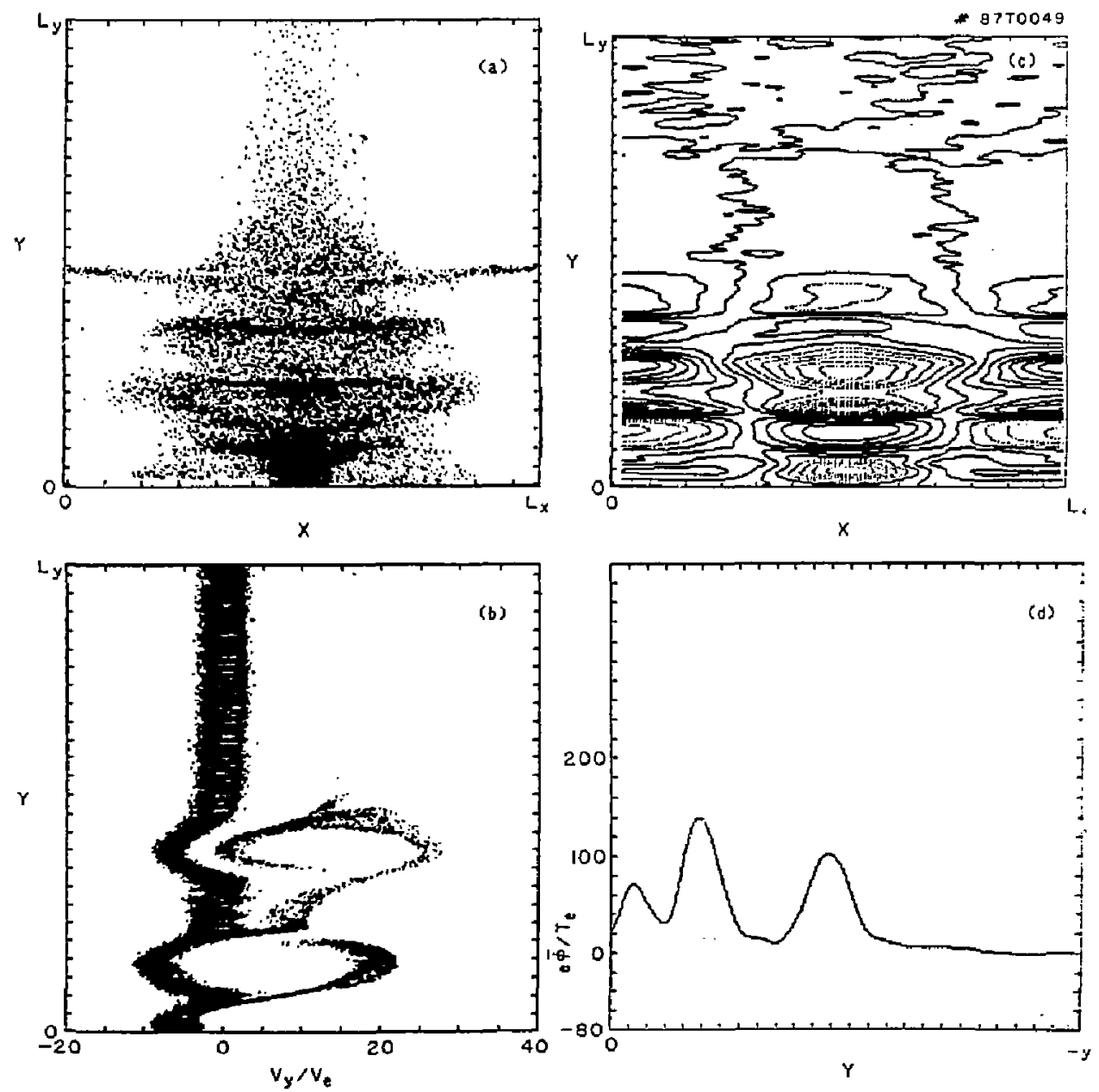

Fig. 12 

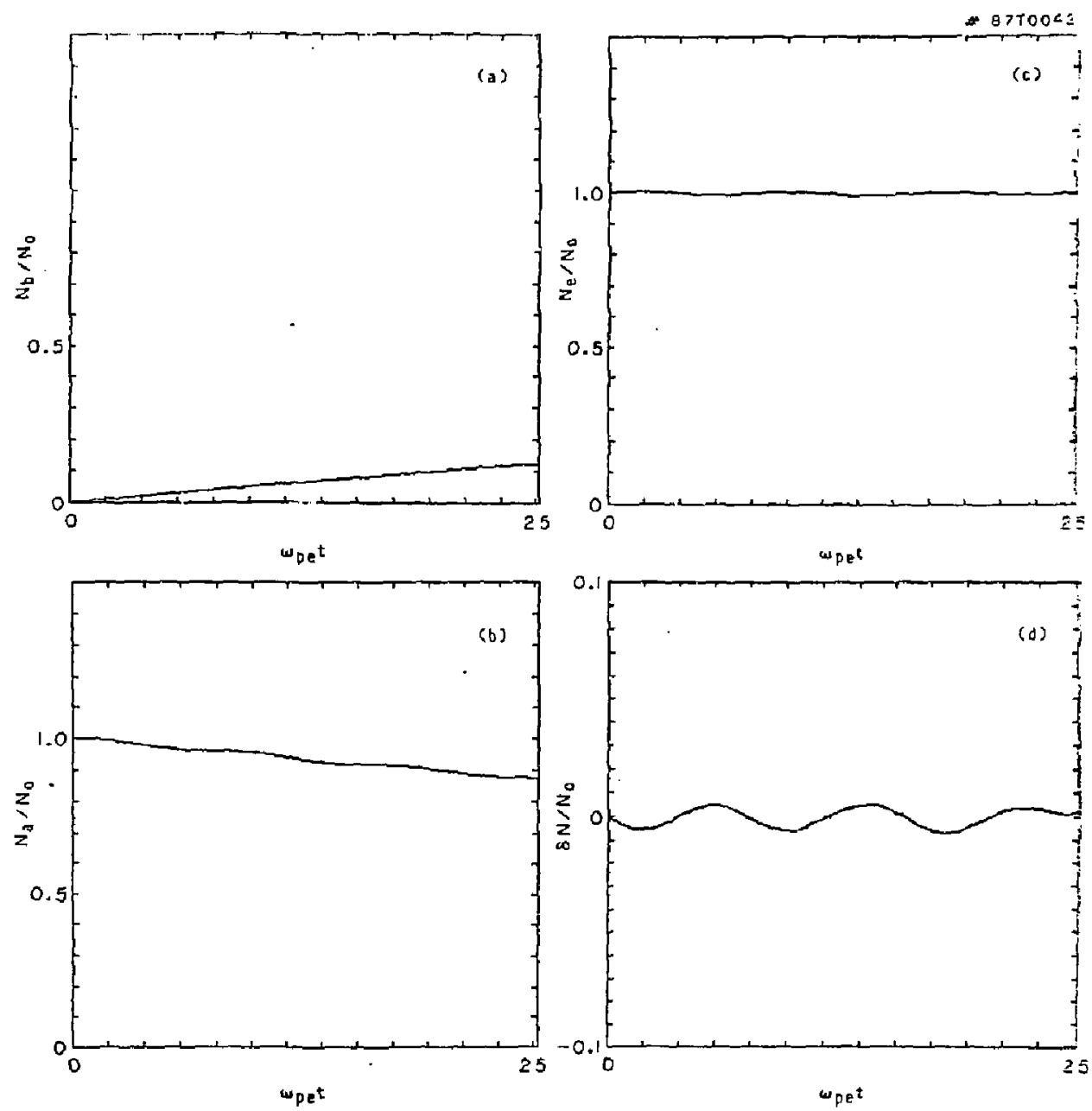

Fig. 13 

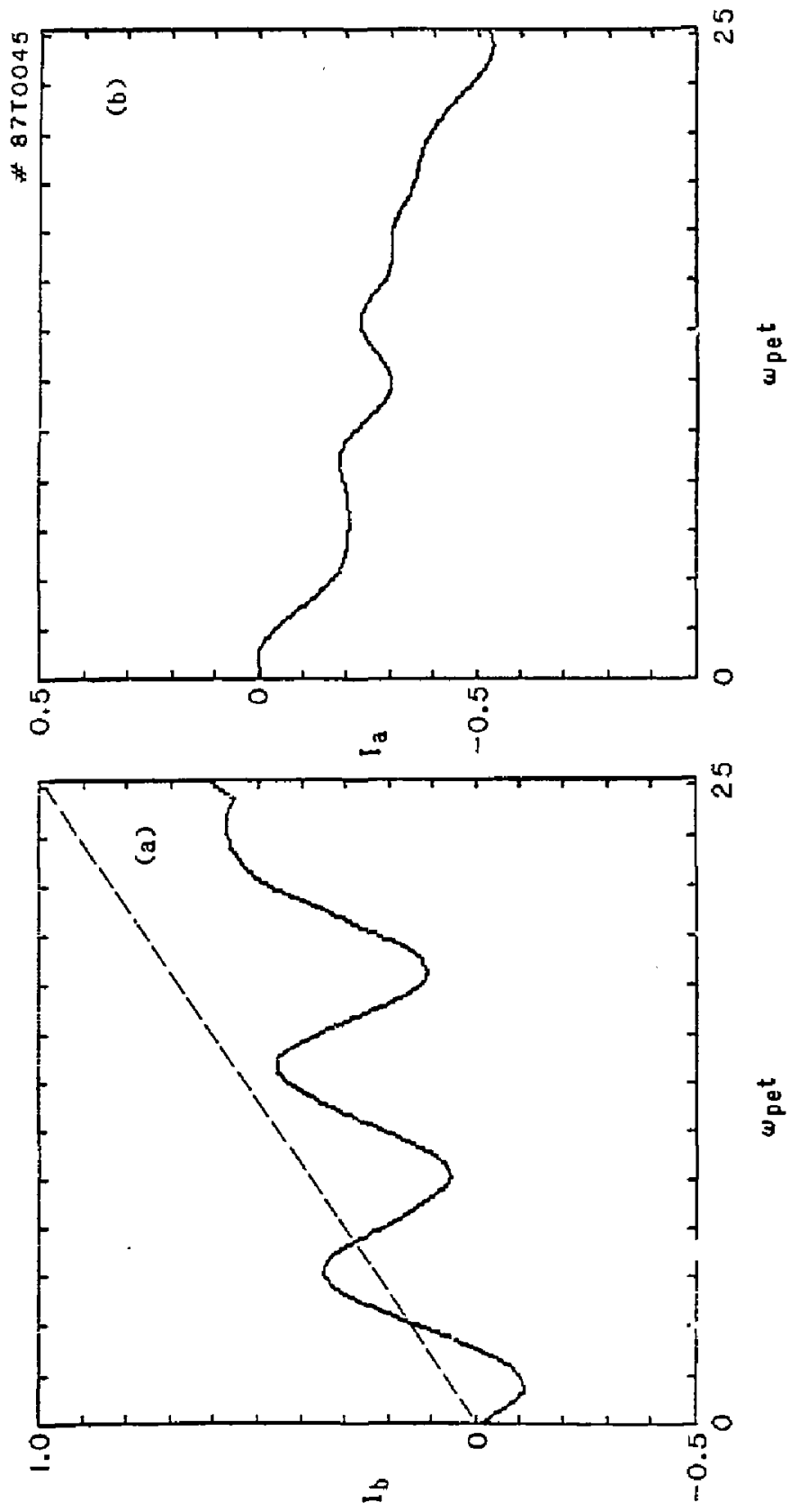

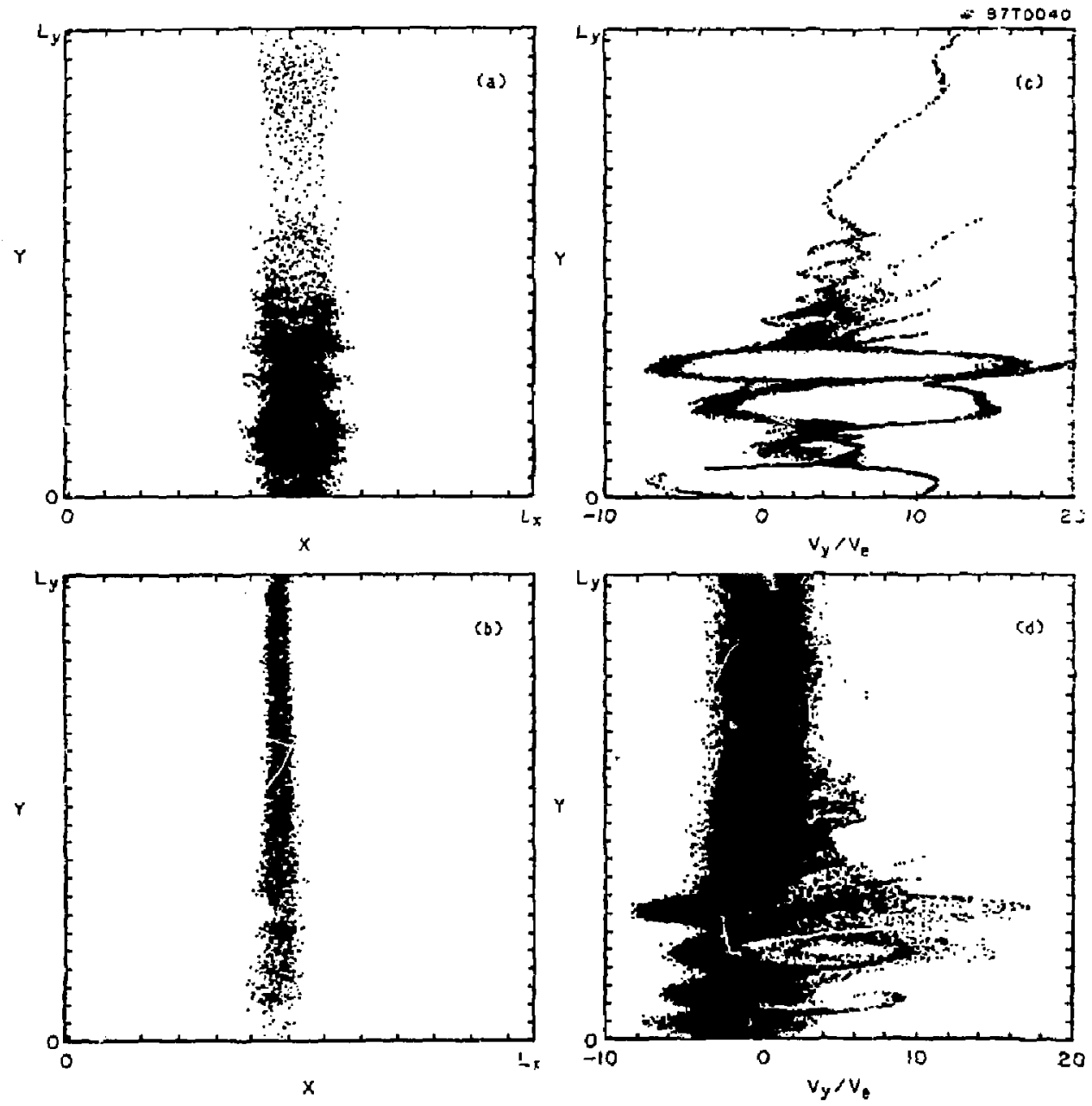

Fig. 15 

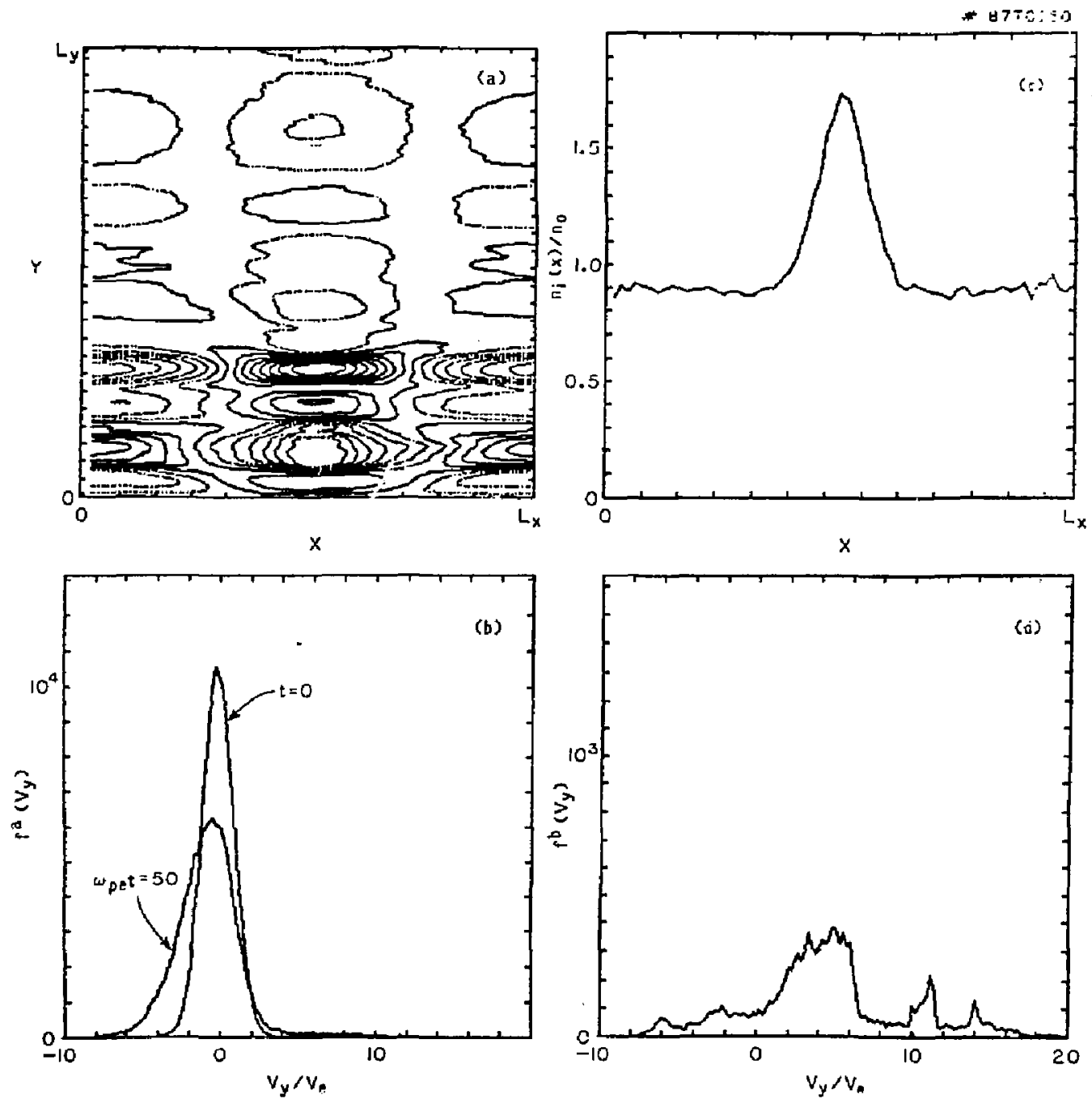

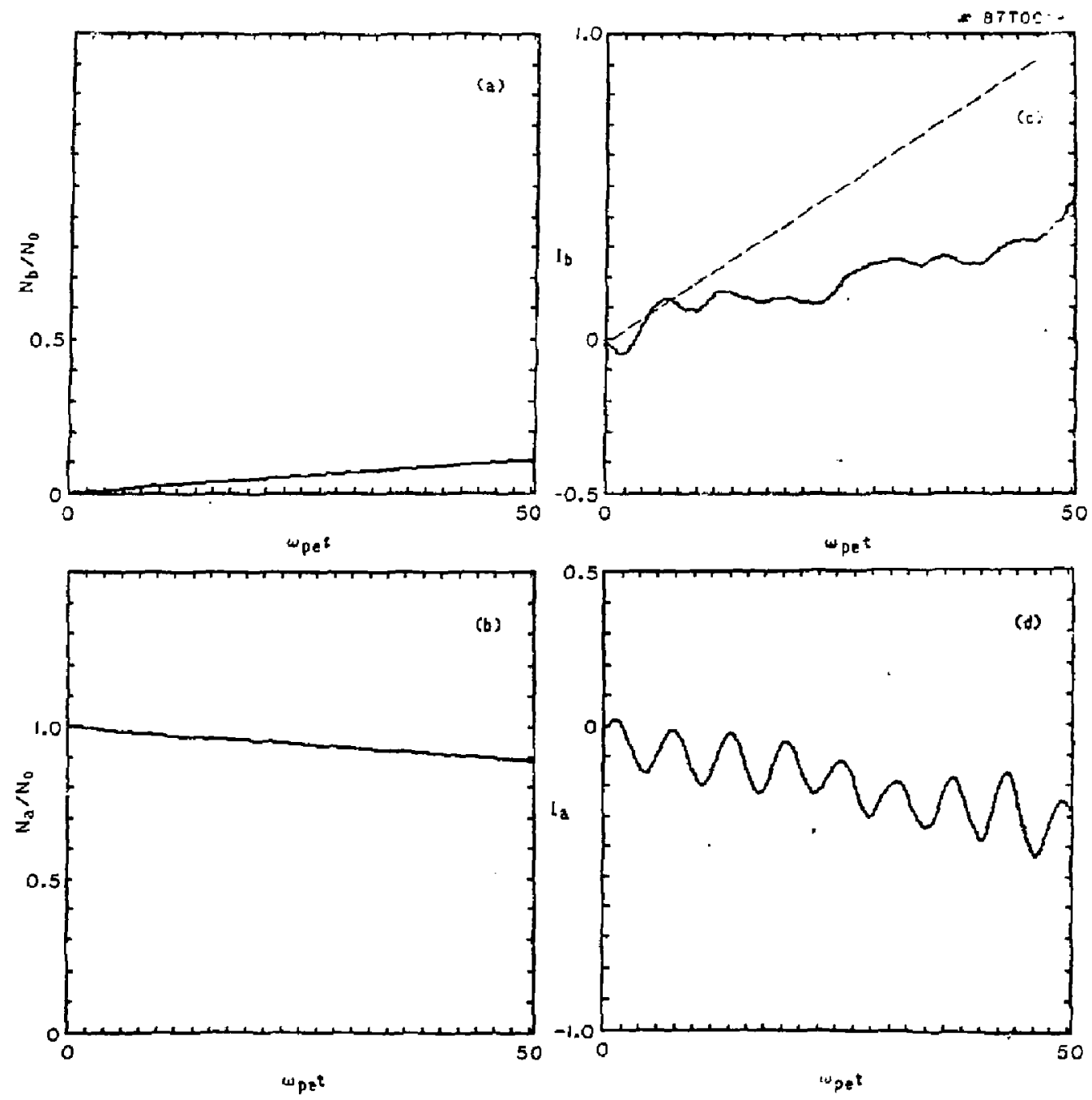

Fig. 17 

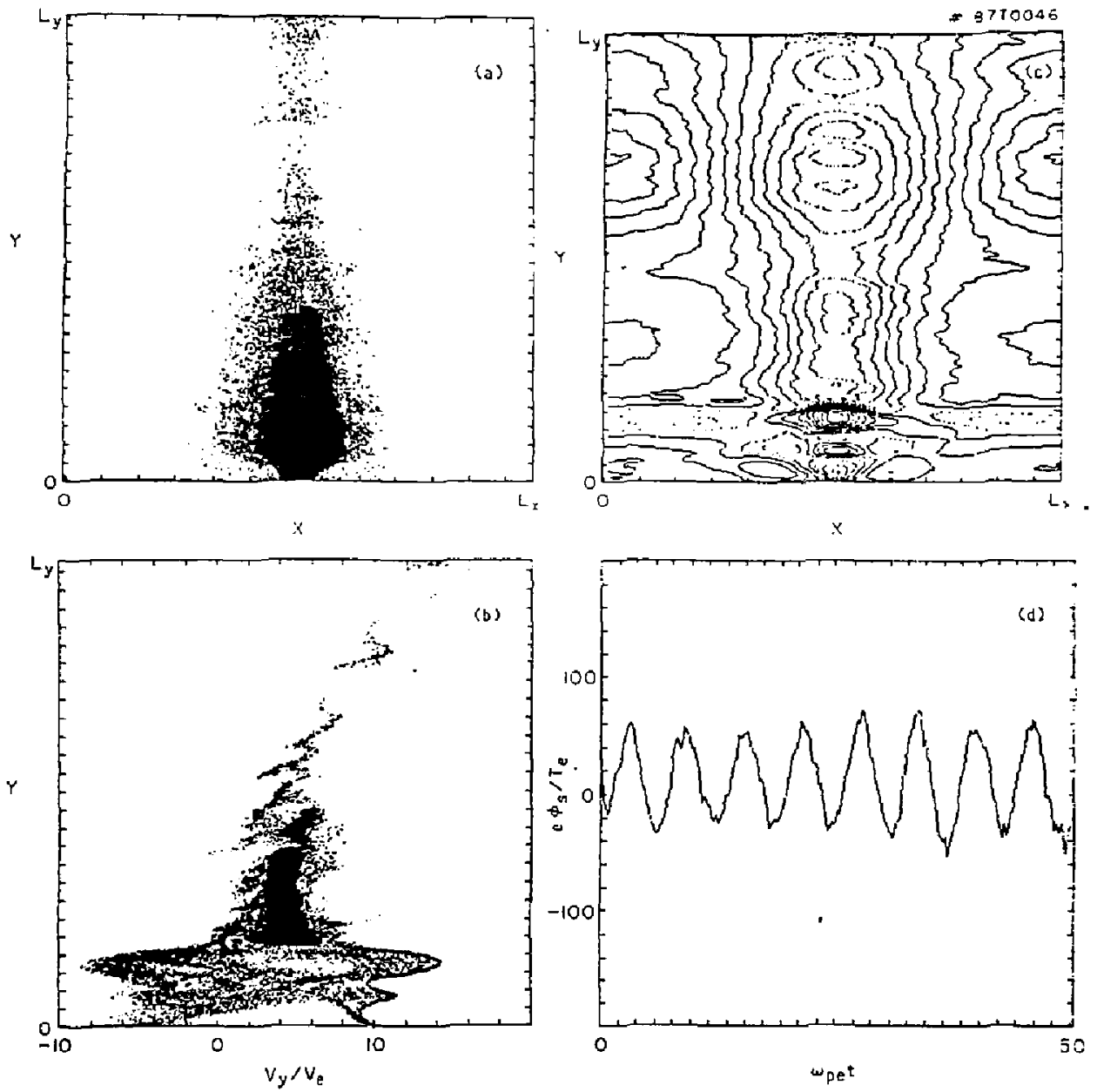

Fig. 18 

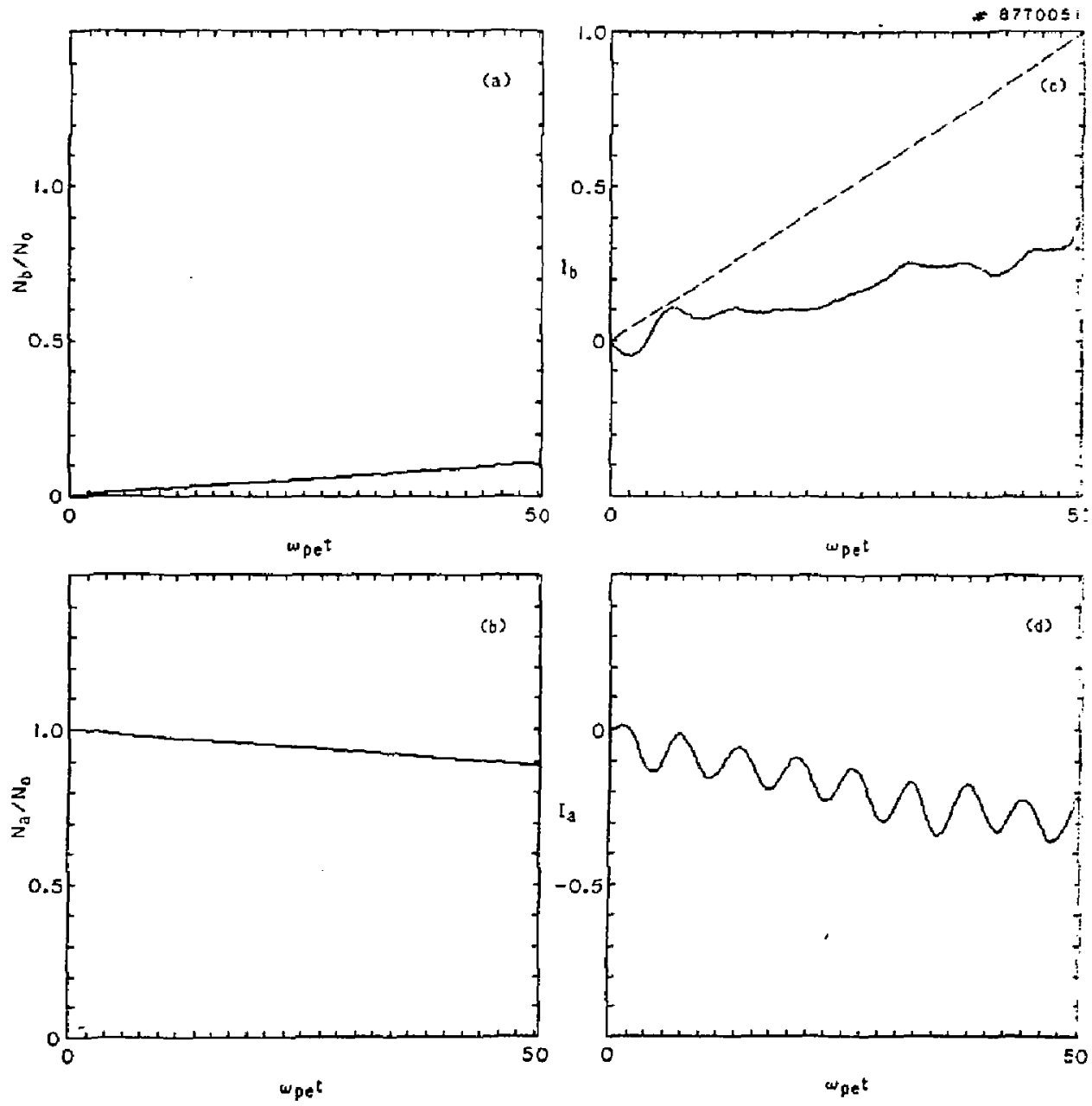

Fig. 19 
Dr. Frank J. Paolonl, Jniv of wollongong, AUSTRALIA Prof. Mom. Bromnan, ijniv Syoney, Australia

Plasma Research Lab., Australian Nat. UnIv., AUSTRALIA

Prof. I.R. Jones, filinders Univ.., NuSTRal IA

Prof. F. Cap, Inst Theo Phys, AusialA

Prof. M. Helndlar, Instltut fur Theorotisehe Physlk, AUSTRIA

M. Goossons, Astronomischi InstItuut, BELGIUM

Ecole Royale Mllitaire, Lab de Phys Plasmas, BELGILM

Con. of European, Dg XII Fusion Prog, BELGIUM

Prot. R. Bouclque, Laboratorling voor Natuurkunda, BELGiluM

Dr. P.H. Sakangka, UnIy Estadual, ERAZIL

instituto De Posquisas Espaciasl-INPE, BRAZIL

Library, Atomle Energy of Canado LInlted, CANADA

Or. M.P. Bachynskl, ipg Technologles, InC.. CANADA

Or. H,, , 5karsgard, Unly of Saskatcheman, CANAOA

Or. H. Barnard, Unlyorslty of BrItlsh Coluabla, CANAOA

Prof. J. Talchmann, Unlve of Montreal, CANMDA

Prot. S.R. Sreenivasan, Univorsity of Calcary, GANADA

Prot. Tudor W. Johnston, INRS-Enorglis, CANADA

Dr. C.R. James, ItnIV. of Alberta, CrIIADA

Dr. Puter Lukac, Komenskoho UAlY, CZECHOSLOVAKIA

The Librartan, Culinam Laboratory, EMGLANO

Mrs. 5.A. Hutchinson, JET Library, ENGLAND

C. Mouttot, Lab. de Physlque dos MIII eux Ionlses, FRAHCE

J. Radot, CEM/CADARACHE - Bat 506, FRAHCE

Or. Ton Mual, Acadeny BIbllographle, hONG KONG

Preprint Library, Cont Ros Inst Phys, HuNGARY

Dr. B. Dasgupta, sahs Inst, IMOIA

Dr. R.K. Chnajlanl. VIkran Univ. INolA

Dr. P. Kaw, Instltuto tor Plasma Rescoreh, INO|A

Or. Phlll to Rosenau, Israel inst Toch, ISRAEL

Prof. 5. Cuparman, Tal AvIV University, ISRAEL

Librarian, int'l Cor Theo Phys, ITALY

Praf. G. Rostagni, UnIv ol Padova, ITAly

MIss Clella Do Palo, Assoc EURATOH-ENEA, ITALY

Blblioteca, del ONR EURATOM, ITALY

Dr. H. Yamato, Toshiba Res \& Dev, JAPAN

Prof. 1. Kawskami, Atomle Enargy Res. Institute, JAPAN

Prot. Kyojl Nishikawa. Univ of HIroshima, JAPAW

Direc. Dept, Lg. Tokamok Res. JAERI, JAPAN

Prat. Satoshl Itoh, Kyushu Unlvarslty, JAPAN

Research Info Center, Nagoya Unlversity, JAPAN

Prof. S. Tanaka, Kyoto University. JAPAN

Library, Kyoto UnIverslty, JAPAN

Prof. NobuyukI Inoue, University ot Tokyo, SNPAK

5. Mor 1, JAERI, JAPAN

M.H. Kim, Koree Advanced Energy Pasearch Instltute, KOREA

Prof. D.1. Chol, Ady. Inst 5cl \& Tech, KOREA

Prof. B.S. LIley, UnIvarsity of Walkato, NEW ZEALAND

Institute of Plasma Physles, PEOPLE'S REPU⿴LIC OF CHINA

Liorarian, institute of Phys.., PEOPLE'S REPUALIC OF CHINA

Library, Tsing Hua Unlversity, PEOPLE'S REPU⿴L冂) $1 C$ OF CHINA
Z. LI, SOuthwest Inst. Physles, PEOPLE'S REPLELIC OF CHINA Prat. J.A.C. Cabral, Inst Superlor TOCA, POATUGal

Dr. Detavian Petrus, AL I CUZA University, ROMANIA

Dr. Johan de villlers, Plasma Physles, AEC, SO AFRICA

Prot, $M_{+} A$. Hall loarg, Untvarsity of Natal, SO AFRICA

Fusion Oiv. LIbrary, JEN, SPAIN

Or. Lennart Stentlo, IinIvarsity of UMEA, SHEDEN .

LIbrary, Royal Inst Toch, SHEDEN

Prof. Hans withalmson, Chalmars univ Toch, SWEDEN

Cantre Phys des Plasmas, Ecol. Polytoch Fod, SWITZERLAND

Blbllotheek, Fom-1 nst Yoor PIasma-fysles, THE NETHERLANDS

Dr. D.D. Ryutor, SIberian Acad Scl, USSR

Dr. G.A. ElIseew, Kurchator institute, USSR

Or. V.A. Glukhlkh, Inst Electro-Physical, USsR

Dr. V.T. Tolok, Inst, Phys. Tech, USSR

Or. L.M. Kavrlzhnykh, institute Gen. Physles, USSR

Prot. T.J.M. Boyd, Unir Colloge H Walos, WALES

Nucloor Res. Establishment, Julleh Ltd., W. GERMANY

Bibllothak, Inst, Fur Plaseatorschung, W. GERMNY

Dr. K. SchIndler, Ruhr UnIversitat, W. GERMANY

AsOEX Raading Fin, IPP/Max-P! lanek=Instl tut fur

Plasmaphysik, W. GERManY

Ltbrorlan, Moxplonck Institut, W. GERMAY

Prot, R.K. Janov, Inst Phys, YUSOSLAVIA 